\title{
Genetic factors contributing to extensive variability of sex-specific hepatic gene expression in Diversity Outbred mice
}

\author{
Tisha Melia $\odot$, David J. Waxman ${ }^{*} *$ \\ Department of Biology and Bioinformatics Program, Boston University, Boston, Massachusetts, United States \\ of America \\ *djw@bu.edu
}

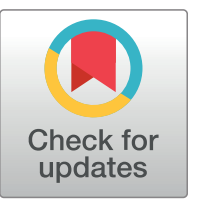

\section{G OPEn ACCESS}

Citation: Melia T, Waxman DJ (2020) Genetic factors contributing to extensive variability of sexspecific hepatic gene expression in Diversity Outbred mice. PLOS ONE 15(12): e0242665. https://doi.org/10.1371/journal.pone.0242665

Editor: Gregory S. Barsh, HudsonAlpha Institute for Biotechnology, UNITED STATES

Received: November 1, 2020

Accepted: November 9, 2020

Published: December 2, 2020

Copyright: @ 2020 Melia, Waxman. This is an open access article distributed under the terms of the Creative Commons Attribution License, which permits unrestricted use, distribution, and reproduction in any medium, provided the original author and source are credited.

Data Availability Statement: All relevant data are within the manuscript and its Supporting information files.

Funding: Supported in part by National Institutes of Health grant DK121998 (to DJW). The funders had no role in study design, data collection and analysis, decision to publish, or preparation of the manuscript.

Competing interests: The authors have declared that no competing interests exist.

\section{Abstract}

Sex-specific transcription characterizes hundreds of genes in mouse liver, many implicated in sex-differential drug and lipid metabolism and disease susceptibility. While the regulation of liver sex differences by growth hormone-activated STAT5 is well established, little is known about autosomal genetic factors regulating the sex-specific liver transcriptome. Here we show, using genotyping and expression data from a large population of Diversity Outbred mice, that genetic factors work in tandem with growth hormone to control the individual variability of hundreds of sex-biased genes, including many long non-coding RNA genes. Significant associations between single nucleotide polymorphisms and sex-specific gene expression were identified as expression quantitative trait loci (eQTLs), many of which showed strong sex-dependent associations. Remarkably, autosomal genetic modifiers of sex-specific genes were found to account for more than 200 instances of gain or loss of sexspecificity across eight Diversity Outbred mouse founder strains. Sex-biased STAT5 binding sites and open chromatin regions with strain-specific variants were significantly enriched at eQTL regions regulating correspondingly sex-specific genes, supporting the proposed functional regulatory nature of the eQTL regions identified. Binding of the male-biased, growth hormone-regulated repressor BCL6 was most highly enriched at trans-eQTL regions controlling female-specific genes. Co-regulated gene clusters defined by overlapping eQTLs included sets of highly correlated genes from different chromosomes, further supporting trans-eQTL action. These findings elucidate how an unexpectedly large number of autosomal factors work in tandem with growth hormone signaling pathways to regulate the individual variability associated with sex differences in liver metabolism and disease.

\section{Introduction}

Sex differences in mammalian gene expression are not limited to reproductive tissues, but also occur somatic tissues [1], most notably the liver, as seen in mouse [2-4], rat [5, 6] and human $[7,8]$. Sex-differential gene expression impacts hundreds of genes that control sex differences in liver function, including metabolism of drugs [9-11], other xenobiotics [12, 13] and fatty 
acids [14], as well as sex differences in liver disease [7, 15-18]. Sex differences in the liver transcriptome characterize protein-coding transcripts $[6,19]$, long noncoding RNAs (lncRNAs) $[20,21]$ and miRNAs [22-24]. Growth hormone (GH) is a key regulator of sex-biased gene expression $[25,26]$ and acts via its sex-dependent pattern of pituitary secretion: pulsatile in males and nearly continuous in females in both rodents and humans [27-29]. These sex differential plasma GH profiles, in turn, induce the sex-differential activation of the JAK2/STAT5 signaling pathway in hepatocytes. STAT5 and downstream GH-dependent transcription factors bind to individual sites in liver chromatin in a sex-biased manner, resulting in sex-differences in liver transcription [30-32]. Sex differences are also evident in the liver epigenome, including extensive sex differences in open chromatin regions (DNase I hypersensitive sites; DHS) [33, 34], chromatin marks [4, 35, 36], DNA methylation [37-39] and 3D genomic interactions and organization [40] associated with the transcription of sex-specific genes.

Genetic modifiers can contribute to the regulation of sex-biased genes. In one early example, male-biased expression of $C y p 2 d 9$ is abolished by a single nucleotide polymorphism (SNP) in the 5' regulatory region [41]. In another example, the Regulator of Sex-Limitation (Rsl) gene represses certain sex-specific genes in a strain-specific manner [42, 43]. Genetic polymorphisms can impart individual differences in drug-metabolizing enzymes [44], many of which are encoded by sex-specific genes [11,45]. Genome-wide association studies linking genetic variants and liver gene expression have identified many expression quantitative trait loci (eQTLs) for liver-expressed genes [46-50]. Little is known, however, about the extent to which such genetic factors impact individual variability in sex-biased gene expression. Understanding the source of variability in sex-biased gene expression in liver has far-reaching implications, as sex-biased genes can impact liver diseases with sex differences in susceptibility, severity and in some cases etiology [51], including non-alcoholic fatty liver disease (NAFLD) [52] and hepatocellular carcinoma [53,54], and polygenic dyslipidemia and coronary artery disease $[7,55,56]$.

Here, we investigate the impact of genetic regulation on sex-specific gene expression using Diversity Outbred (DO) mice, an outbred population derived from eight inbred mouse strains $[57,58]$. DO mice have high natural allelic variance $[59,60]$ and diverse phenotypes $[61,62]$, which facilitates fine genetic mapping of liver-specific traits. Full genomic sequences are available for all eight DO founder strains [63], allowing genotypes in individual DO mice to be mapped back to specific founder strains. Using this model, we find unexpectedly high rates of gain and loss of sex-specific gene expression across DO founder mouse strains. We identify novel autosomal eQTLs targeting thousands of liver-expressed genes, including 1,500 eQTLs regulating liver-expressed lncRNA genes. Almost 1,000 of the eQTLs we identified target sexspecific genes, and many of these show sex-dependent genetic associations that explain the gain or loss of sex-specific gene expression in DO founder mice. Further, we link sex-specific binding of GH-regulated transcription factors and sex differences in chromatin accessibility to eQTL regions regulating sex-specific genes, and we identify co-regulated gene clusters defined by their overlapping eQTLs, a subset of which involve trans regulation. This elucidation of genetic factors controlling liver sex differences has important implications for our understanding of inter-individual variability in sex-biased liver function and disease.

\section{Results}

\section{High variability of sex-specific gene expression across DO mouse founder strains}

We identified 1,033 protein-coding genes that show sex-specific expression in mouse liver in one or more of nine mouse strains examined (418 male-biased genes and 615 female-biased 
genes). These strains include CD-1 mice, used in our earlier studies of sex-biased liver gene expression [34], and the eight founder strains used to establish the DO mouse population (A/J, C57BL/6J, 129S1/SvlmJ, NOD/ShiLtJ, NZO/HILtJ, CAST/EiJ, PWK/PhJ, WSB/EiJ) [57, 60]. Sex-biased gene expression was highly variable across the eight DO founder strains (Fig 1A), with sex bias lost in at least one strain for $96 \%$ of the genes examined, and only 20 genes showing sex-biased expression in all 8 strains (see full dataset in S1 Table; examples are shown in S1 Fig). This strong strain dependence also characterized the most highly sex-specific genes, with sex-biased expression lost in at least one strain for 58 of 74 genes with a sex-specific expression ratio $>4$ at FDR $<0.05$. Finally, analysis of the 220 sex-specific genes with the highest interstrain variability revealed expression patterns highly specific to each strain, further confirming the striking variability of sex-specific gene expression in DO founder mouse livers (Fig 1B).

The number of sex-specific genes identified in each founder strain ranged 2.4-fold, from 77 genes (strain A/J) to 183 genes (strain PWK/PhJ; S2A Fig, S1 Table). A similar pattern was seen when only the most highly sex-specific genes were considered (S2B Fig). Principal component analysis (PCA) based on the expression levels of all sex-specific genes revealed that the largest variance (PC1) corresponds to the sex of each liver, as expected, while the second component (PC2) clustered the individual liver samples based on the evolutionary distance between strains (Fig 1C). Thus, the six Mus musculus domesticus subspecies strains are closer to each other than to the other subspecies strains, i.e. Mus musculus castaneus (strain CAST/ EiJ) and Mus musculus musculus (strain PWK/PhJ). Further, among the Mus musculus domesticus strains, the lone, wild-derived WSB/EiJ strain was separated from the other five strains, recapitulating the published phylogenetic tree shown in Fig 1C [64].

We implemented DAVID analysis $[65,66]$ to determine whether the sex-specific genes identified in each strain showed differential enrichment for particular biological pathways. Sixteen KEGG pathways were enriched (adjusted $\mathrm{P}<0.05$ ) in at least one of the eight sets of sexspecific genes examined (Fig 1D). Six pathways were significantly enriched in nearly all strains, including cytochrome P450-catalyzed drug and lipid metabolism, identifying this as a conserved sex-specific biological function, while ten pathways showed strain-specific enrichments (see y-axis dendrogram). Pyruvate metabolism was uniquely linked to sex-specific genes in WSB/EiJ livers, while biosynthesis of unsaturated fatty acids was most strongly enriched in $\mathrm{PWK} / \mathrm{PhJ}$ livers.

\section{Impact on GH regulatory mechanisms}

Our finding that some mouse strains contain comparatively few sex-specific genes, e.g., A/J and CAST/EiJ mice (S2 Fig), raises the possibility that these strains might be deficient in one of the four major classes of GH-regulated sex-specific genes found in liver [30, 67]. These classes of sex-specific genes are defined based on their responses to surgical removal of the pituitary gland by hypophysectomy, which ablates pituitary secretion of $\mathrm{GH}$, as well as secretion of all other pituitary-dependent hormones. Class 1 male-specific and female-specific genes are activated in liver by the pituitary GH secretion profile in livers of the sex where they are more highly expressed, and consequently, following hypophysectomy, class 1 genes are down regulated in the sex where they show higher expression. In contrast, class 2 male-specific and female-specific genes are repressed in liver by the GH secretion profile of the sex where they are less highly expressed, and consequently, following hypophysectomy, class 2 genes are up regulated (de-repressed) in the sex where they show lower expression. We did not observe any major differences in the distribution of sex-specific genes across the four GH/hypophysectomy response classes in any of the DO founder strains, as compared to the distribution in CD-1 mouse liver (Fig 1E). Thus, the major GH-dependent regulatory mechanisms for liver sex- 
A.

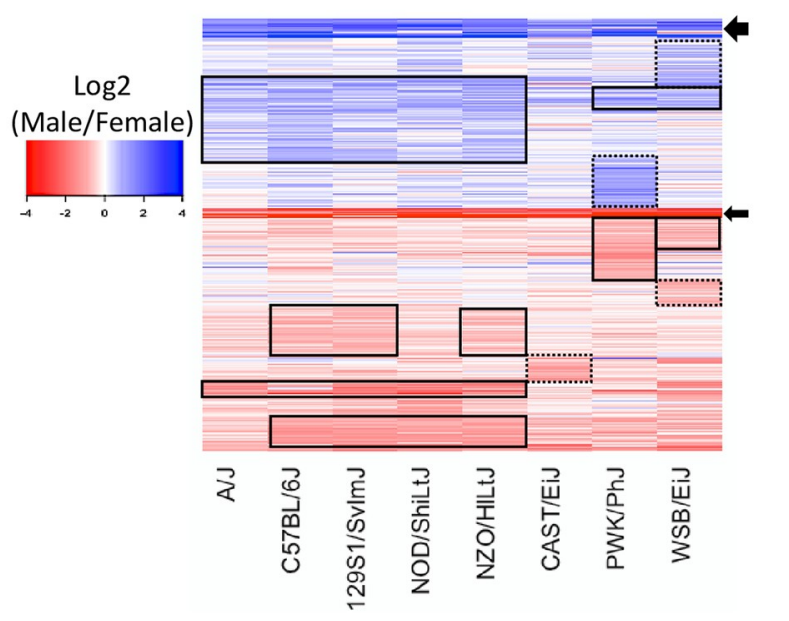

B.

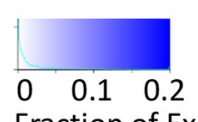

Fraction of Expression

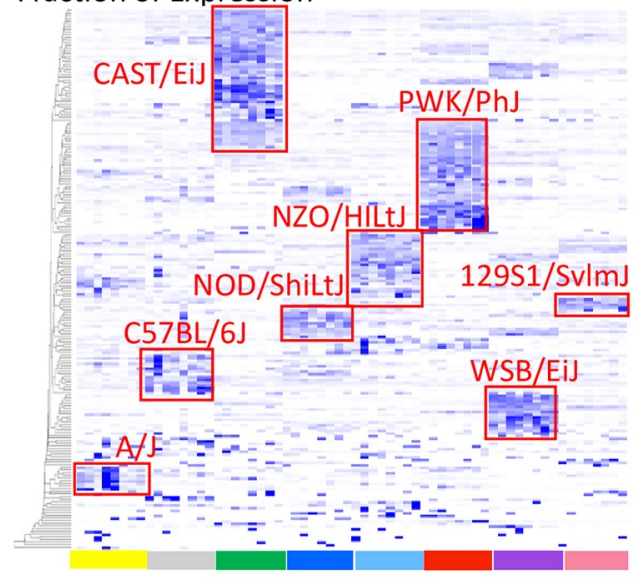

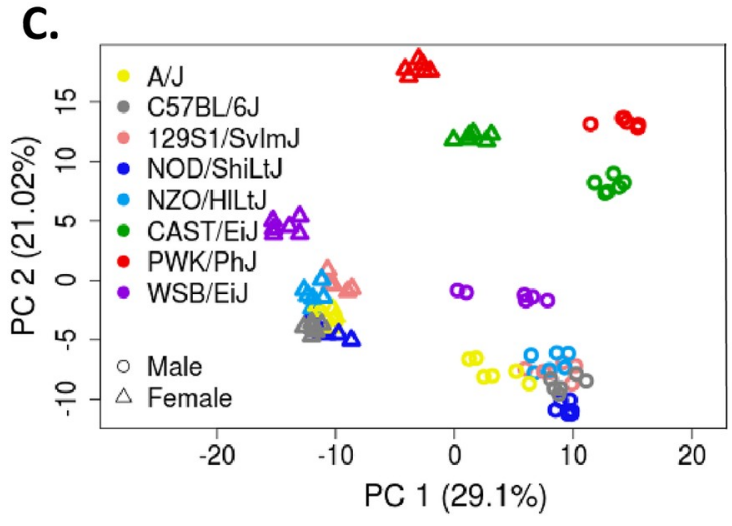

D.

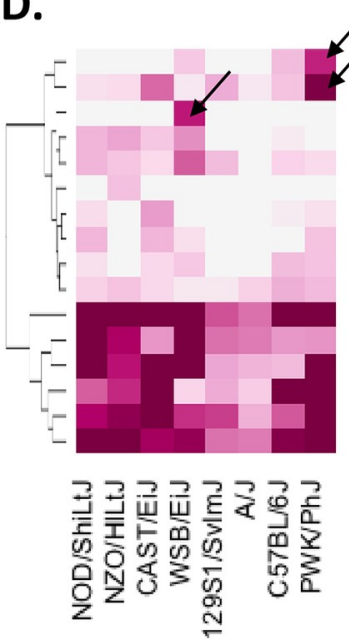
Fatty acid degradation PPAR signaling pathway Amoebiasis

Glutathione metabolism Fatty acid elongation

Retinol metabolism

Metabolic pathways

Arachidonic acid metabolism

Chemical carcinogenesis

Steroid hormone biosynthesis
Biosynthesis of unsaturated fatty acids Metabolism of xenobiotics by cytochrome P450 Pyruvate metabolism

Inflammatory mediator regulation of TRP channels

Complement and coagulation cascades

Drug metabolism - cytochrome P450

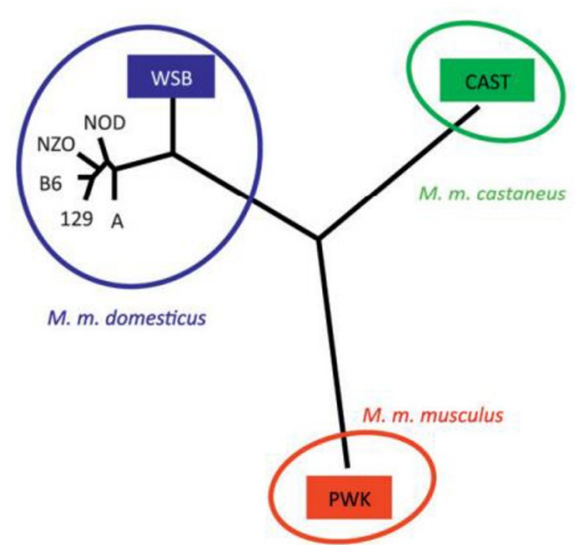

E.

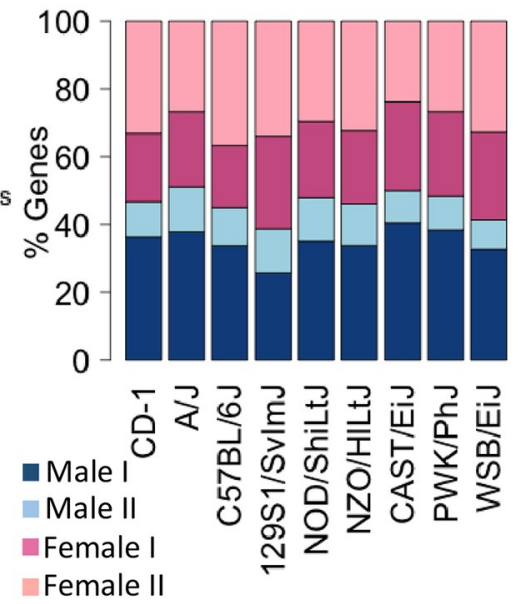

Fig 1. Sex-specificity across DO founder strains. (A) Heatmap of log2 male/female fold-change values across DO founder mouse livers for 471 protein-coding genes that show sex-biased expression (male/female |fold-change| $>1.5$ at FDR $<0.05$ ) in at least one DO founder strain based on microarray analysis. Solid black boxes, genes with strong sex-specificity in the indicated strains; dashed boxes, strong sex-specificity is limited to one strain. Arrows, strong sex-biased expression in all 8 strains. Sex-specific expression was lost in at least one of the 8 strains for 451 of these 471 genes. (B) Gene expression level for 220 sex-specific genes with the highest ratio of inter-strain variability to intra-strain variability (see Methods) across DO founder mouse livers. The map was clustered based 
on the summation of expression levels of each gene across the 8 indicated DO founder strain male livers, with the color intensity indicating the fraction of expression seen in each liver. Data shown are based on 8 male livers for each DO founder strain. (C) PCA of 96 microarray datasets (6 livers/sex/strain) based on the expression level of 471 sex-specific genes in each DO founder strain. Shown at the right is a phylogenetic tree [64] of the eight DO founder strains based on the SNP data for chr11. The three DO founder strains whose names are in colored boxes are the more distant wild-derived inbred strains, which matches the more distant patterns of sex-specific gene expression seen in the PCA plot. (D) KEGG pathways enriched (adjusted Benjamini P $<0.05$ ) in the set of sexspecific protein-coding genes identified in each DO founder mouse strain. (E) Distribution of male-specific class 1 and class 2 genes, and of female-specific class 1 and class 2 genes across mouse strains. Each bar presents the distribution of sex-specific genes across four hypophysectomy response classes identified earlier [30].

https://doi.org/10.1371/journal.pone.0242665.g001

biased gene expression captured by the hypophysectomy class 1/class 2 gene designations are apparently preserved across the DO founder strains, despite high variability in the expression seen for many individual sex-specific genes.

\section{eQTLs for liver-expressed genes}

We analyzed published genotyping and expression datasets from 438 individual DO mouse livers $[46,68,69]$ (S2 Table) to discover eQTLs representing significant associations between $\mathrm{SNPs} /$ indels and the expression of either protein coding genes or multi-exonic liver-expressed lncRNA genes [20,21]. This analysis was performed using the full set of 438 livers, and separately, all male livers $(n=219)$ or all female livers $(n=219)$ (S3A Fig). We identified 10,325 significant autosomal eQTLs when considering all livers, 7,414 autosomal eQTLs when considering male livers only, and 8,233 autosomal eQTLs when considering female livers only, giving a total of 12,886 autosomal eQTLs (Table 1, Fig 2A). The total number of eQTLs increased to 17,278 were found when chrX eQTLs were included (S3 and S4A Tables). Half of the autosomal eQTLs identified $(6,344$ out of 12,886$)$ were not identified in earlier studies [ 46 , $48-50,69$ ] and are novel, and include a large majority of the 1,524 eQTLs associated with liver-expressed lncRNA genes [20]. More autosomal eQTLs were identified in female than in male liver (Table 1), similar to findings in BXD mouse liver [49], suggesting genetic regulation is more common for the female liver transcriptome. In striking contrast, male DO liver eQTLs on chrX were 8.4 times more frequent than female DO liver eQTLs (3,932 vs. 468 eQTLs; S3A Table). This latter finding is consistent with the enrichment of sex-interacting eQTLs on human chrX [70], and with the observation that X-linked genetic variation in humans is significantly greater in males than in females [71]. Finally, 66\% of autosomal eQTLs are within the

Table 1. Number of autosomal eQTLs found when using all, male only or female only DO mouse livers. The number of genes associated with each set of eQTLs is shown in parenthesis. Last column: number of Combined eQTLs found in the same TAD as the genes they are associated with. More significant eQTLs were found in female than male DO mouse liver, similar to BXD mouse liver, where 1,638 significant eQTLs were found in male liver vs. 2,076 significant eQTLs in female liver (Gatti DM et al, 2010). See S4A Table for full listing of eQTLs.

\begin{tabular}{|c|c|c|c|c|c|c|}
\hline & Set of genes & All Livers & Male Livers & Female Livers & Combined & Cis eQTLs \\
\hline & & \multicolumn{5}{|c|}{ number of eQTLs (number of genes) } \\
\hline \multirow[t]{3}{*}{ All eQTLs } & $\begin{array}{l}\text { Protein-coding } \\
(\mathrm{n}=18,543)\end{array}$ & $\begin{array}{l}9,088(8,753 \\
\text { genes) }\end{array}$ & $\begin{array}{l}6,494(6,202 \\
\text { genes) }\end{array}$ & $\begin{array}{l}7,219(6,904 \\
\text { genes })\end{array}$ & $\begin{array}{l}\begin{array}{c}11,362(10,097 \\
\text { genes })\end{array} \\
\end{array}$ & $\begin{array}{l}7,492 \\
(66 \%)\end{array}$ \\
\hline & LncRNA $(\mathrm{n}=2,016)$ & $\begin{array}{c}1,237(1,177 \\
\text { genes) }\end{array}$ & 920 (885 genes) & 1,014 (957 genes) & $1,524$ ( 1,318 genes $)$ & $\begin{array}{l}1,014 \\
(67 \%)\end{array}$ \\
\hline & Total eQTLs: & 10,325 & 7,414 & 8,233 & 12,886 & 8,506 \\
\hline \multirow[t]{3}{*}{$\begin{array}{l}\text { eQTLs associated with } 1,196 \text { sex-specific } \\
\text { genes }\end{array}$} & $\begin{array}{l}\text { Protein-coding } \\
\quad(\mathrm{n}=1,033)\end{array}$ & 708 (682 genes) & 555 (523 genes) & 602 (581 genes) & 830 (743 genes) & $631(76 \%)$ \\
\hline & LncRNA $(\mathrm{n}=163)$ & 131 (121 genes) & 99 (95 genes) & 105 (97 genes) & 157 (130 genes) & $108(69 \%)$ \\
\hline & Total eQTLs: & 839 & 654 & 707 & 987 & 739 \\
\hline
\end{tabular}

https://doi.org/10.1371/journal.pone.0242665.t001 
A.

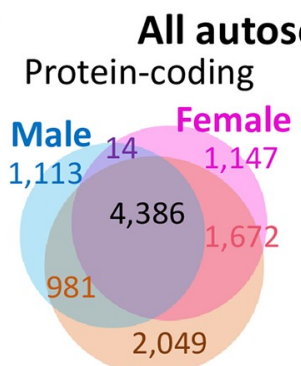

All

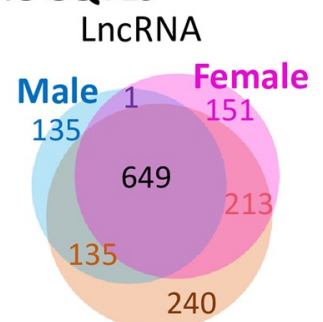

All
B.

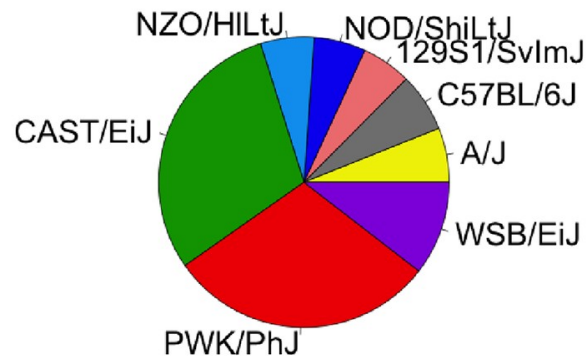

C. Sult3a1 All livers

Male livers

Female livers
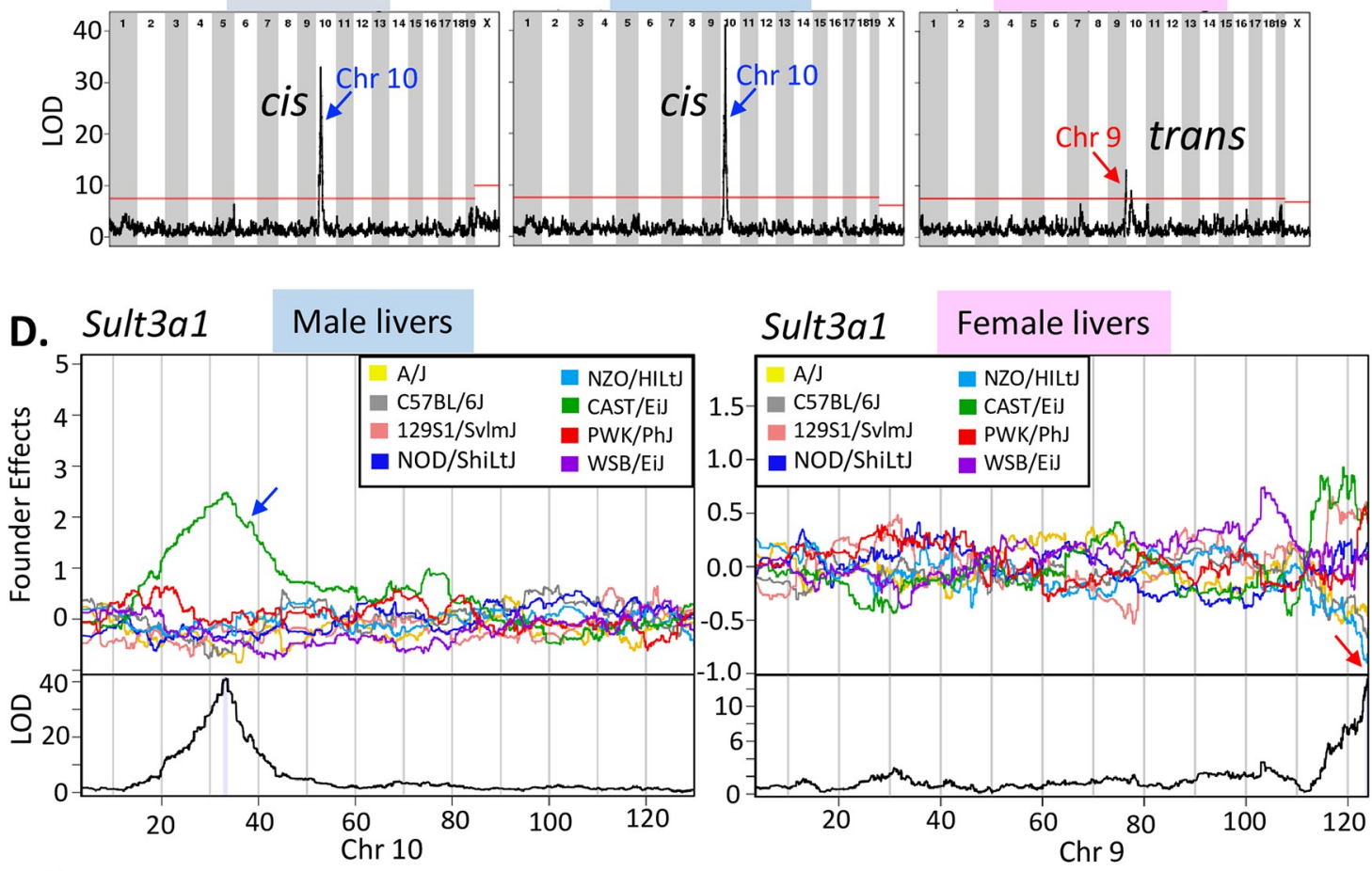

E.
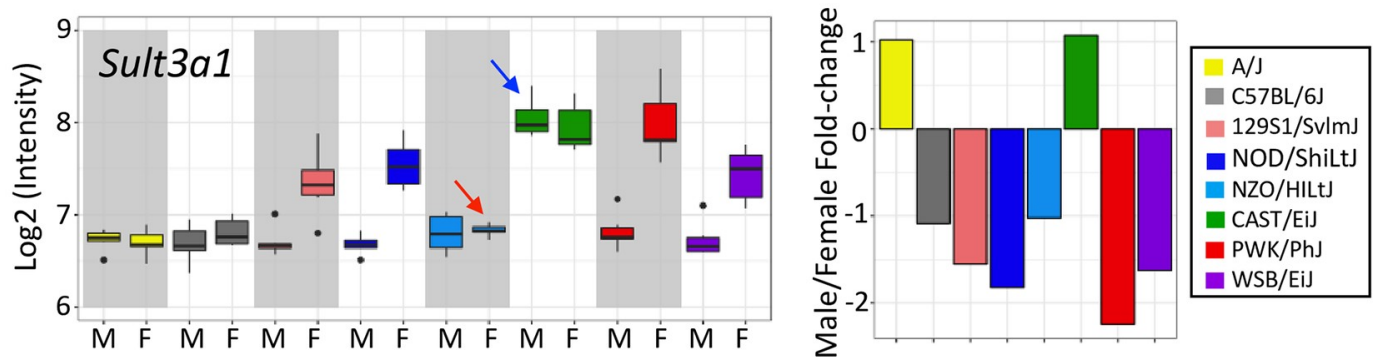

Fig 2. Autosomal eQTLs for liver-expressed genes. (A) Venn diagrams showing numbers of autosomal eQTLs discovered using all DO livers, or using male only or female only liver samples and that are associated with protein-coding genes (left) or lncRNA genes (right). Overall, 10,597 autosomal eQTLs are significant in either male liver or female liver (Table 1). (B). Pie chart showing the realtive frequency at which each of the indicated DO founder strains has the largest regression coefficient (i.e., is the major regulating strain) for the combined set of 12,886 autosomal eQTLs. (C) Genome-wide association of Sult3a1 in all, male only, and female only DO livers. The horizontal red line marks the $\mathrm{P}<0.05$ significance cutoff based on the permutation test. (D) Regression coefficients (top of each panel) and LOD scores (log10(p-value); bottom of each panel) across the chromosome that has a significant eQTL peak in male (left) or female (right) mouse liver, as marked at bottom. Shaded area in the LOD score plot indicates the 95\% Bayesian credible interval for each eQTL. (E) Expression of Sult3al in male and female mouse livers for each DO founder strain (left). Also 
shown are calculated male/female expression ratios (right). The middle hinge of each boxplot indicates the median, and the lower and upper hinges indicate the first and third quartiles. Whiskers mark $1.5^{*} \mathrm{IQR}$ value. Red arrows indicate a decrease in female liver expression in NZO/HiLtJ mice and an increase in male liver expression in CAST/EiJ mice, both of which abolish the sex-specific expression seen in the respective mice strain.

https://doi.org/10.1371/journal.pone.0242665.g002

same TAD [72] as the genes they are associated with, consistent with genetic regulation in cis (Table 1). In contrast, all 4,392 eQTLs on chrX were trans-acting (S4A Table).

For each eQTL, we identified the founder strain whose SNP/indel is most likely responsible for the genetic association captured by the eQTL (the 'regulating strain'). This was indicated by the founder strain whose regression coefficient at the SNP marker where the strongest association occurs (i.e. the SNP marker with the highest LOD score) had the largest absolute value (S4A Table). $60 \%$ of all autosomal eQTLs discovered using all DO livers (6,158 of 10,325 eQTLs) were associated with genetic variants in either CAST/EiJ or PWK/PhJ mice (Fig 2B; see specific example in Fig 2C-2E). Those two strains are the most evolutionarily divergent (Fig 1C) and have the largest number of SNPs and indels [60]. The WSB/Eij strain follows (10\% all of autosomal eQTLs), followed by the other five founder strains ( $6 \%$ of autosomal eQTLs each), highlighting how liver gene expression patterns diverged as genetic variants accumulated during evolution. A majority (54-67\%) of all autosomal eQTLs were associated with up regulation of gene expression in the regulating strain (S3B Fig), and 36\% (3,706 of 10,325 autosomal eQTLs) were associated with changes in expression in two or more regulating strains (S4A Table, column AC).

\section{Genetic regulation of sex-specific genes}

eQTLs regulate a large fraction of sex-specific genes, with 873 of 1,196 sex-specific genes (73\%) being associated with a total of 987 autosomal liver eQTLs (Table 1, Fig 3A). 143 of these eQTLs were only significant when using male livers (66 eQTLs) or only when using female livers (77 eQTLs) (S4A Table). Further, many of the eQTLs discovered using both male and female DO livers showed a stronger genetic association in one sex (S4B Table, S4 Fig). eQTLs that are stronger in male DO livers were biased for association with strongly male-specific genes, and those that are stronger in female liver were more closely associated with strongly female-specific genes, as indicated by the distributions of their sex-differential LOD scores (Fig 3B). eQTLs within the top $10 \%$ of LOD score differences between male and female livers (top 1,060 of all 10,597 such autosomal eQTLs; S4A Table) were enriched 5.1-fold for association with genes of matched-sex-specificity, as compared to genes with the opposite sex-specificity (P-value $<6.5 \mathrm{e}-07$, Fisher's Exact test). Multiple eQTLs were identified for 199 sexspecific genes (S4C Table), indicating these genes are subject to multiple, distinct forms of regulation. Finally, 240 sex-specific genes were not associated with any eQTLs (S4D Table). These expression of these 240 genes across DO livers was less variable than that of sex-specific genes with eQTLs, as was seen in both male DO $(\mathrm{P}<0.01$, Student's $\mathrm{t}$-test $)$ and female DO livers $(\mathrm{P}<0.007$, Student's t-test); average expression levels were similar for both gene sets $(\mathrm{P}>0.28$, Student's t-test).

Next, we investigated whether eQTLs with a sex-bias in their association can explain the variability in sex-specific gene expression seen in DO founder strains. We examined 360 autosomal eQTLs for sex-specific genes that are significant in either male only or female only DO liver samples (or both) and are associated with either an increase or a decrease in sex-specificity in the regulating strain (S5 and S6 Tables). These associations encompass 322 (68\%) of the 471 sex-specific protein-coding genes for which expression data is available for all eight founder strains in both sexes. We identified four circumstances whereby an eQTL decreases 


\section{A Sex-specific autosome eQTLs B}

Sex-specific Sex-specific Protein-coding

Male 5 Female Male > Female

55

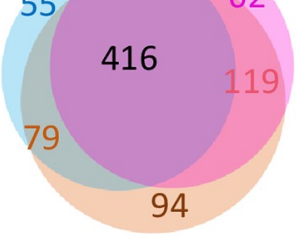

All

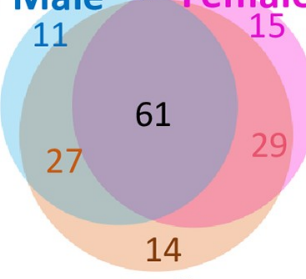

All

Sex-independent Male-specific Female-specific
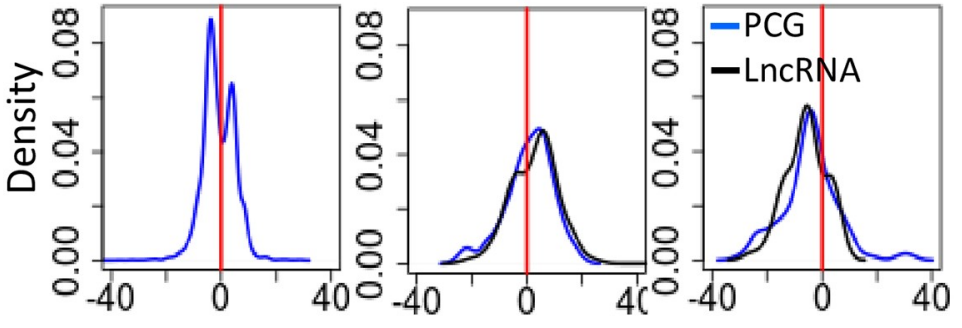

LOD Male - LOD Female

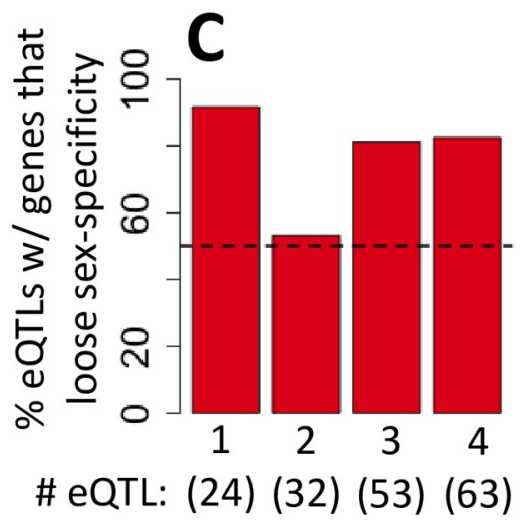

Male Liver

\begin{tabular}{c|l}
$\begin{array}{c}\text { Stronger } \\
\text { eQTL in } \\
\text { Male } \\
\text { liver }\end{array}$ & $\begin{array}{l}\text { Repression of male- } \\
\text { specific gene }\end{array}$ \\
\cline { 2 - 2 } & Up regulation of \\
female-specific gene
\end{tabular}

Female Liver

Stronger Up regulation of eQTL in male-specific gene 3 Female

liver
Repression of femalespecific gene

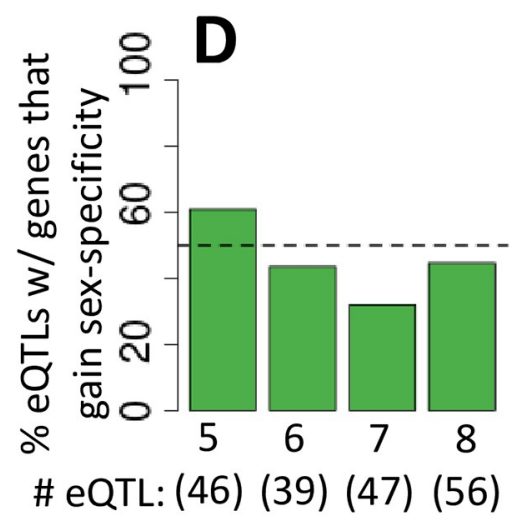

Male Liver

\begin{tabular}{c|l|}
$\begin{array}{c}\text { Stronger } \\
\text { eQTL in } \\
\text { Male } \\
\text { liver }\end{array}$ & $\begin{array}{l}\text { Up regulation of } \\
\text { male-specific gene }\end{array}$ \\
\cline { 2 - 2 } & $\begin{array}{l}\text { Repression of } \\
\text { female-specific gene }\end{array}$
\end{tabular}

Female Liver

Stronger Repression of maleeQTL in specific gene Female liver
Up regulation of female-specific gene

Fig 3. Autosomal eQTLs associated with sex-specific genes. (A) Venn diagrams showing overlaps for numbers of eQTLs discovered in all, male only or female only DO liver samples that are associated with sex-specific protein-coding or sex-specific, multi-exonic intergenic lncRNA genes. (B) Distribution of LOD score differences (log10 values) in male vs. female liver for autosomal eQTLs that are associated with genes that are sexindependent (left), strongly male-specific (male/female |fold-change| $>4$; middle) or strongly female-specific (male/female $\mid$ fold-change $>4$; left). (C) (Left) Distribution of 172 eQTLs in categories \#1-4 (described at the right), which are associated with a loss of sex-specific gene expression in the regulating strains. (Right) Four scenarios, whereby an eQTL deceases the sex-specfiicity of gene expression. (D) (Left) Distribution of 188 eQTLs in categories \#5-8 (described at the right), which are associated with a gain of sex-specific gene expression in the regulating strains. (Right) Four scenarios, whereby an eQTL increases the sex-specificity of gene expression. 
the sex-specificity of expression in the regulating strain (Fig 3C; 172 eQTLs in categories \# 1-4), and four other circumstances whereby an eQTL increases sex-specificity in the regulating strain (Fig 3D; 188 eQTLs in categories \# 5-8) (S6 Table, S5 Fig), where a decrease or an increase in expression is inferred from the regression coefficient in the regulating strain (S5 Table). One example is the category \# $2 \mathrm{eQTL}$ on chr10 regulating the female-specific Sult3a1 gene, which is highly significant in male DO livers but was not found in female livers (Fig 2C). This cis eQTL is associated with loss of sex-specificity due to the elevated expression of Sult3a1 in male livers of CAST/EiJ mice, the regulating strain (Fig 2D, left; Fig 2E; blue arrows). Sult3a1 is also associated with a distinct, category \# $4 \mathrm{eQTL}$ on chr9 that was uniquely identified in female livers (Fig 2C). This trans eQTL abolishes sex-specificity due to the decreased expression of Sult3a1 in female livers of NZO/HIltJ mice, its regulating strain (Fig 2D, right; Fig 2E; red arrows). Thus, both eQTLs abolish the sex difference in Sult3a1 expression in their respective regulating founder strains (Fig $2 \mathrm{E}$, arrows), but by different mechanisms.

Overall, 219 of the 360 eQTLs (61\%), impacting 205 sex-biased genes, were associated with either an outright loss or gain of sex-specificity in the regulating strain (S6 Table, column D). For 134 of the 172 (78\%) eQTLs in categories \# 1-4, sex-specific gene expression is lost in the regulating strain but it is retained in at least one other strain. Sex-specific expression is lost in the regulating strain for $81-92 \%$ of eQTLs in categories \# 1,3 and 4 , but for only $53 \%$ of category \# 2 eQTLs (Fig 3C). This indicates that while all category \# 2 eQTLs decrease sex-specificity in the regulating strain, almost half are not strong enough to cause a loss of sex-specificity. Of the 188 eQTLs in categories \# 5-8, only 47\% (85 eQTLs) showed a gain of sex-specificity in the regulating strain (Fig 3D). This suggests there is another layer of gene regulation for many of those genes. These findings highlight the extensive impact and multiple mechanisms through which genetic factors regulate the variability of sex-specific gene expression in DO mice.

\section{Sex-biased regulatory elements within eQTL regions}

Genomic regions encompassed by an eQTL may impact sex-specific gene expression in the regulating founder strain through strain-specific genetic variants at sex-specific regulatory regions, e.g., enhancers. Such regulatory regions include DHS that are sex-biased in mouse liver, i.e., regions where chromatin is significantly more open (more accessible) in one sex, and that harbor sex-biased binding sites for key GH-regulated liver transcription factors [31, 32, 35]. Strain-specific genetic variants at such sites may alter or abolish GH-regulated transcription factor binding activity, and perhaps may alter chromatin accessibility and thereby dysregulate the expression of sex-biased genes. To investigate this hypothesis, we analyzed sexbiased DHS, and separately, sex-biased transcription factor binding sites that contain SNPs/ indels specific to the identified regulating strain within each eQTL region (S4A Table, last columns). Sex-biased DHS with strain-specific variants were enriched at eQTL regions associated with genes with matching sex-specificity, as compared to eQTLs associated with genes showing the opposite sex-bias or genes with no sex-specificity (Fig 4A; first two panels; S6 Fig). The majority of DHS found in any eQTL region, both for sex-biased and sex-independent genes, are sex-independent DHS; these DHS showed a significant enrichment at eQTLs for sex-independent genes, as compared to sex-specific genes (Fig 4A; third panel). A similar pattern of enrichment was found for sex-biased binding sites for the GH-regulated transcription factor STAT5 (Fig 4B). In contrast, male-biased binding sites for the GH-regulated transcriptional repressor BCL6, which preferentially represses female-specific genes in male liver [32, 73], showed significant enrichment at cis eQTL regions for female-specific genes, when compared to eQTL regions for male-specific or sex-independent genes (Fig 4C, first panel). Sex- 


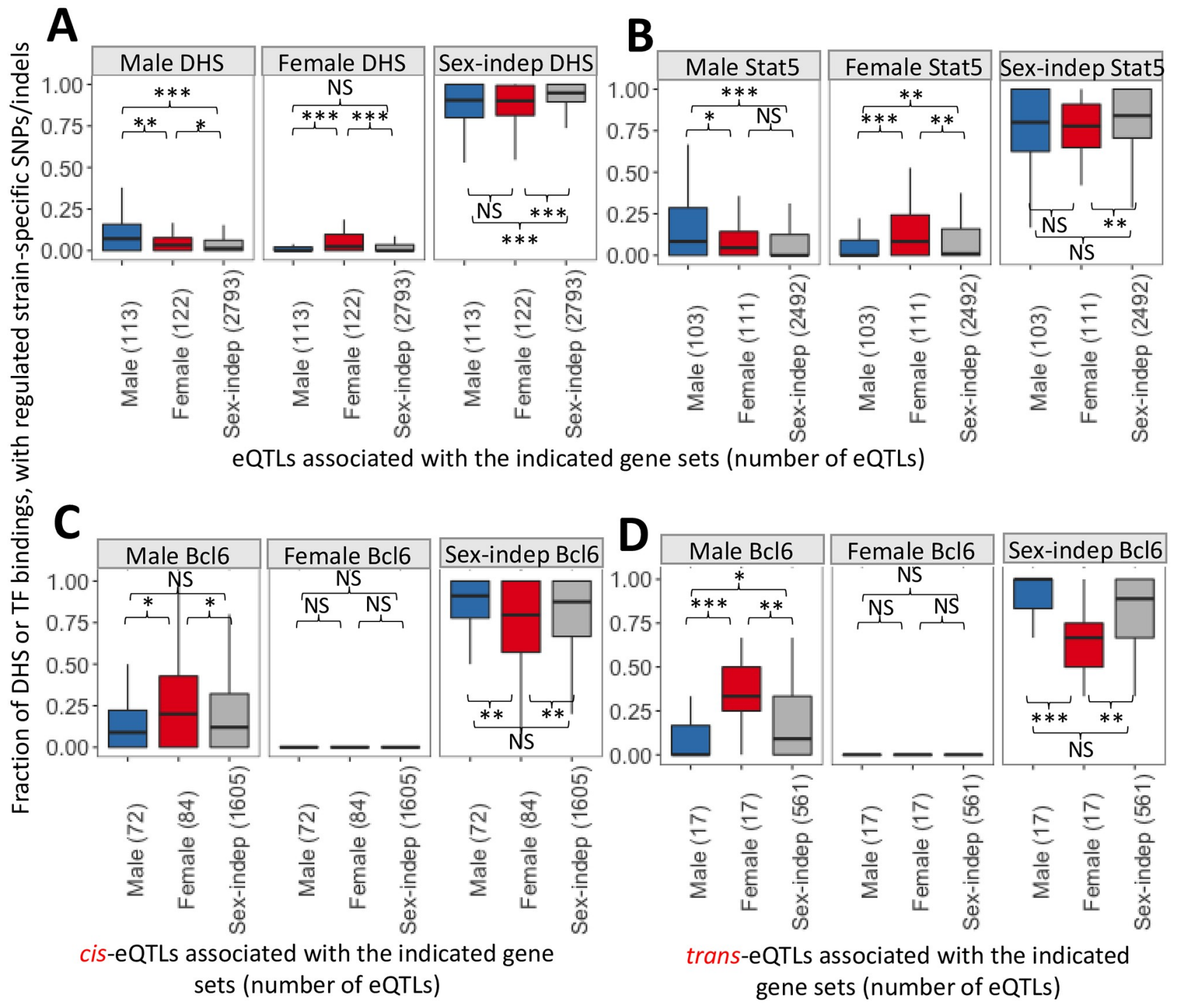

Fig 4. Enrichment at different sets of eQTL regions of sex-biased DHS and transcription factor binding sites that contain regulating strainspecific SNPs/indels. Y-axis, fraction of all DHS (A), or all binding sites for STAT5 (B) or BCL6 (C, for cis-eQTLs; and D, for trans-eQTLs) within the eQTL region that are male-specific, female-specific or sex-independent and that contain (within the DHS or within the binding site) SNPs/indels specific to the regulating strain. This analysis was carried out for the sets of eQTLs for genes expressed in a male-specific, female-specific, or sexindependent manner (sets of eQTLs specified along the $\mathrm{x}$-axis), with the number of eQTLs analyzed in each boxplot indicated in parenthesis. Data shown in A and B are for cis plus trans eQTLs, whereas cis (C) and trans (D) eQTLs were analyzed separately in C and D. The number of DHS and STAT5 or BCL6 binding sites in each eQTL is indicated in S4 Table. For example, each of the three red boxplots in panel D shows the distribution of 17 values, where each value is the fraction of BCL6 binding sites that are male-biased, female-biased, or sex-indepenent (as indicated at top) in a set of 17 trans eQTL regions that are associated with female-specific genes; only BCL6 binding sites that contain SNPs/indels that are specific for the regulated strain are considered. For each factor, the distributions were compared across eQTL sets using the Wilcoxon test $\left({ }^{* * *}, \mathrm{p}<0.001,{ }^{* *}, \mathrm{p}<0.01\right.$ and ${ }^{*}$, $\mathrm{p}<0.05$ ), as indicated in each subpanel. The middle hinge of each boxplot indicates the median value, while the lower and upper hinges correspond to the first and third quartiles. Whiskers marks $1.5{ }^{*} \mathrm{IQR}$ value.

https://doi.org/10.1371/journal.pone.0242665.g004

independent BCL6 binding sites were significantly depleted at eQTL regions associated with female-specific genes (Fig 4C, third panel). Male-biased BCL6 binding sites showed an even greater enrichment at trans eQTL regions (Fig 4D). These enrichments of sex-biased DHS and transcription factor binding sites with strain-specific SNPs/indels provide strong support for the functional relevance of these genomic regions to the eQTL-based regulation. 


\section{Inference of gene co-regulation based on eQTLs}

We hypothesized that genes with overlapping eQTLs are likely to be co-regulated, presumably via shared regulatory regions. Overlapping eQTLs with a common regulating strain were used to define co-regulated gene clusters. We identified 1,521 such co-regulated gene clusters, comprising 4,105 genes, based on eQTLs discovered in all DO liver samples (S7A Table). The vast majority of the co-regulated gene clusters are small in size; $95 \%$ of all clusters include five or fewer genes each, although some clusters contain as many as 25 genes (S7D Table). 28 of the gene clusters were significantly enriched for sex-specific genes (S7A Table). The absence of many large co-regulated gene clusters indicates that strain differences are primarily determined by many individual loci, each regulating a small number of genes, rather than by a small number of variants in master regulatory regions. The gene clusters we identified show evidence of co-regulation, with many genes within clusters showing high pairwise gene expression correlation (see examples in Fig 5). Half (762) of the co-regulated clusters contain subclusters, where the identified eQTL region was associated with up regulation of some genes and down regulation of other genes in the cluster. One example is a 21-gene cluster on chr2, which is predicted to occur in the PWK/PhJ strain (Fig 5A; S7A Table, Cluster100_all). Pairwise gene expression correlation analysis separated these 21 eQTLs into two well-defined subclusters. Eleven overlapping eQTLs in the upper sub-cluster have positive regression coefficients (i.e. increased expression of the 11 genes; S7 Fig) and are all located on the same chromosome (chr2) as their associated genes, whereas 10 overlapping eQTLs in the lower subcluster have negative regression coefficients (i.e., decreased expression of the 10 genes; S7 and S8 Figs) and are each located on a different chromosome than the genes they are associated with. The pairwise gene correlations are less tight in the negatively correlated sub-cluster (e.g. $Z b t b 7 c)$, suggesting the eQTL association may represent indirect regulation or partial regulation involving another factor. Notably, in the case of the eQTL involving $Z b t b 7 c$, an independent, stronger genetic association was identified in cis (chr18, S8 Fig). The co-regulated eQTL cluster in Fig 5A shows that genes from different chromosomes may be grouped together (S7 Fig), highlighting the ability of this approach to identify complex trans regulation. Overall, 159 (10.4\%) of the co-regulated clusters grouped genes from multiple chromosomes. Further, 344 (23\%) of the co-regulated clusters contain both protein-coding and lncRNA genes, generating testable hypothesis for possible functions of the co-clustered lncRNAs. We also found examples of co-regulated clusters containing genes within the same chromosome, indicating local regulatory regions (Fig 5B).

Co-regulated gene clusters based on overlapping eQTLs were also discovered using male only DO livers (S7B Table), and separately, female only DO livers (S7C Table), and clusters with a sex-dependent genetic association were identified. Some of these clusters showed a switch in gene members between clusters identified in male liver as compared to female liver samples. One example involves three female-specific genes from the sulfotransferase gene family: Sult2a1, Sult2a3 and Sult2a5 (Fig 6A). In PWK/PhJ male mouse liver, but not in PWK/PhJ female mouse liver, Sult2a1 and Sult2a5, but not Sult2a3, are predicted to be co-regulated, as their eQTLs overlap and are associated with high expression of both genes (Fig 6B, male samples, top and bottom). In contrast, in CAST/EiJ female liver, Sult2a1 shows co-regulation with Sult2a3, and the high expression of both genes is associated with overlapping eQTL regions (Fig 6B, female samples; top and middle). Even though all three eQTL regions overlap, their eQTL peaks do not overlap, indicating a need for higher resolution genetic mapping to pinpoint the precise regulatory regions and tease apart these two distinct regulations. Sult2a3 and Sult2a5 showed no significant eQTLs in male and female liver samples, respectively (S9 Fig). The pairwise gene correlations between these three genes are consistent with these patterns, as 


\section{A}
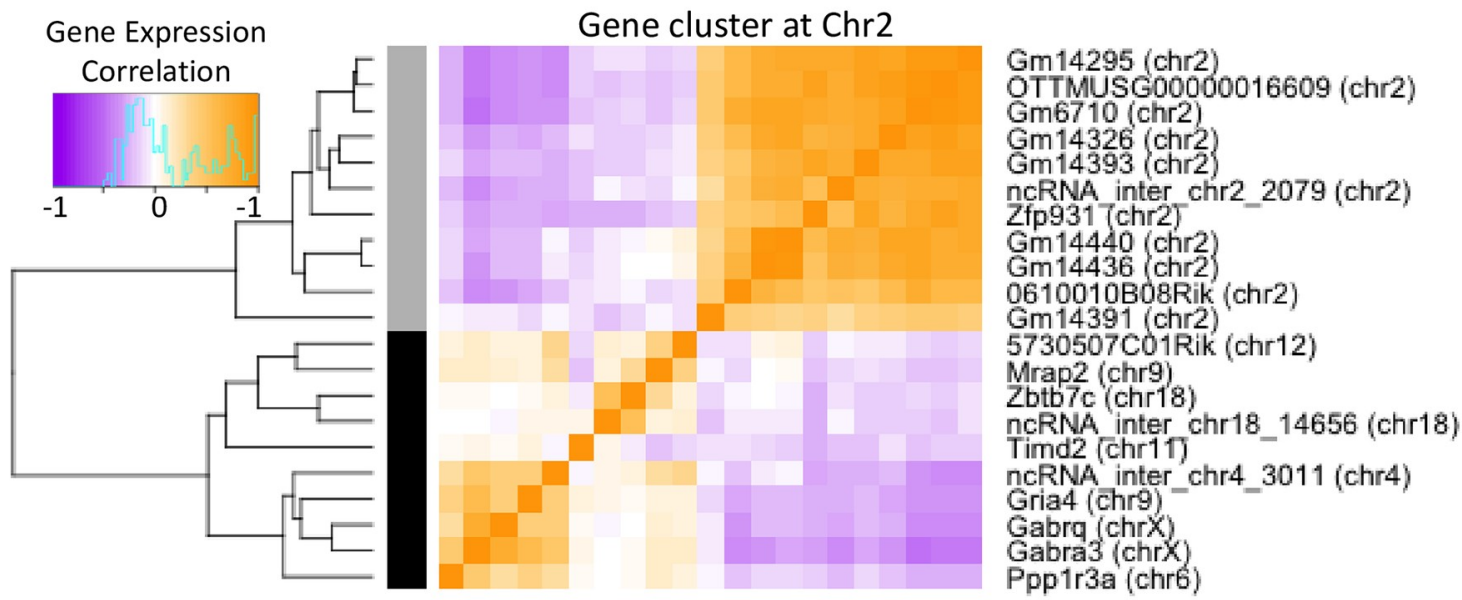

Negative Regression Coefficient

Positive Regression Coefficient

B

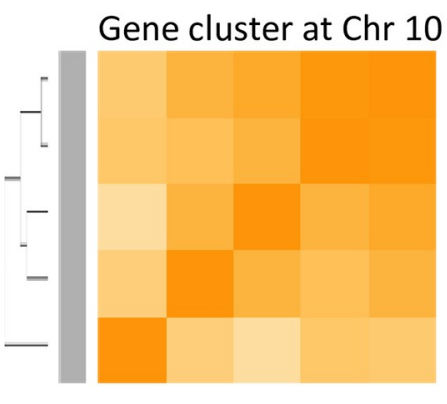

Gm4794 (chr10)

Sult3a1 (chr10)

Clvs2 (chr10)

Xcr1 (chr9)

Dse (chr10)
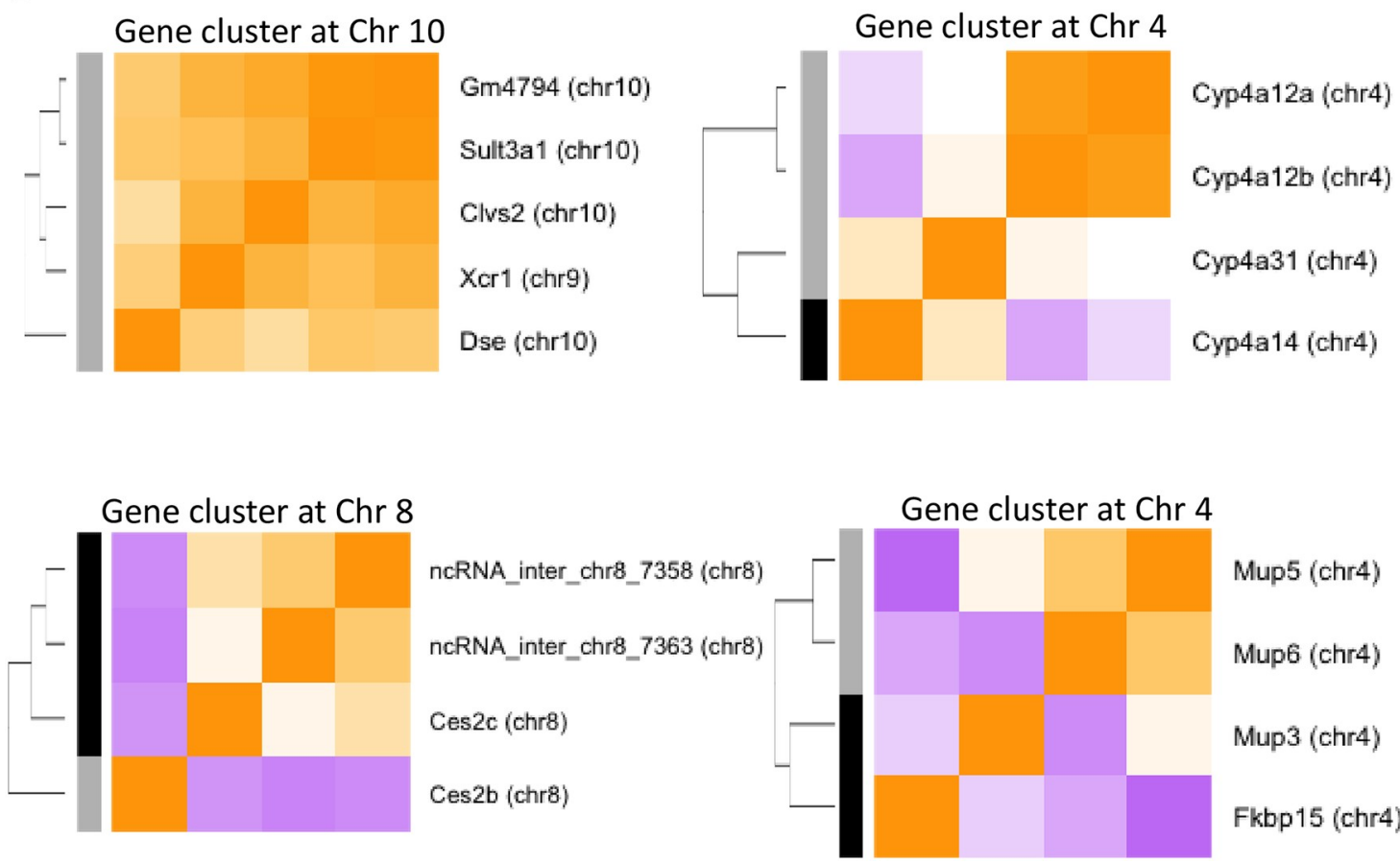

Gene cluster at Chr 4

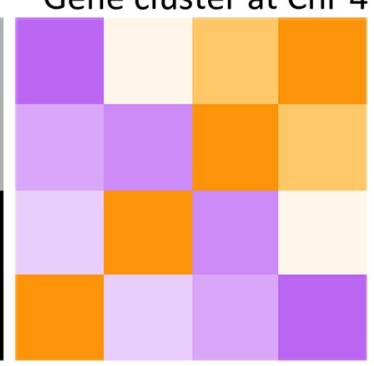

Mup5 (chr4)

Mup6 (chr4)

Mup3 (chr4)

Fkbp15 (chr4)

Fig 5. Co-regulated gene clusters based on overlapping eQTL regions discovered in all liver samples. (A) A co-regulated cluster consisting of twenty-one overlapping eQTLs with the $\mathrm{PWK} / \mathrm{PhJ}$ as the regulating strain. (B) Four co-regulated clusters containing sex-specific genes. The heatmaps show Pearson correlations of gene expression.

https://doi.org/10.1371/journal.pone.0242665.g005 
A. Male liver (strain PWK/PhJ)

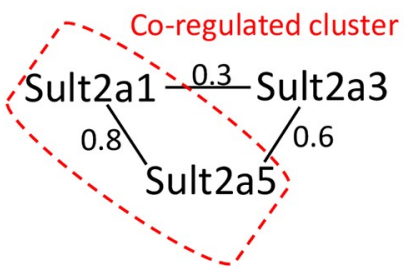

B.
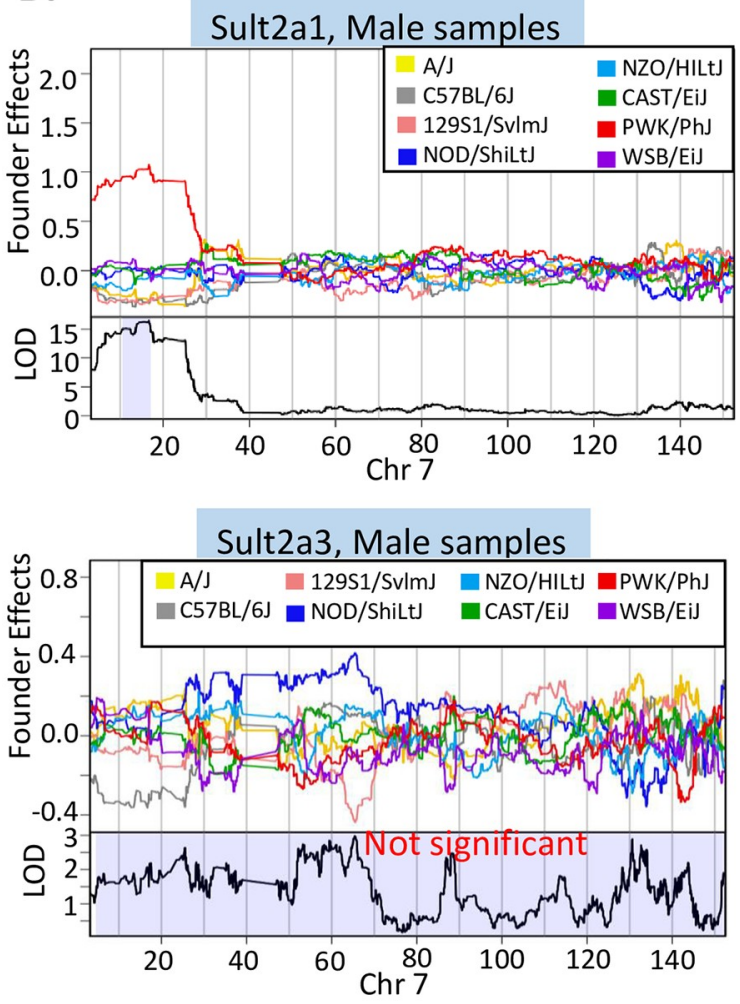

Sult2a5, Male samples

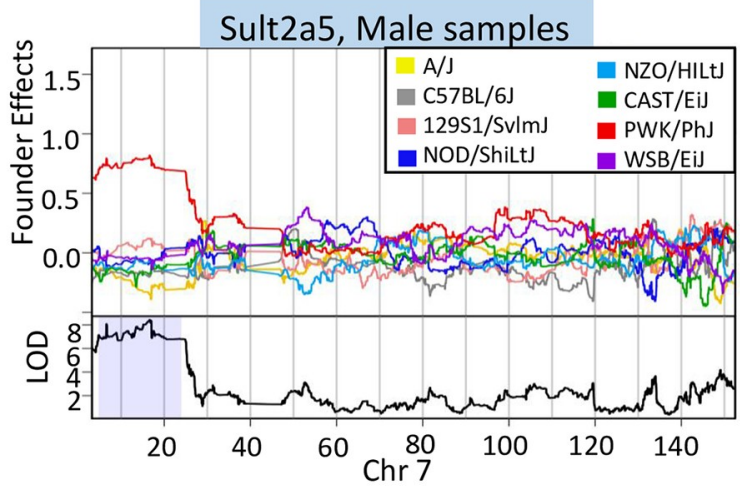

Female liver (strain CAST/EiJ)

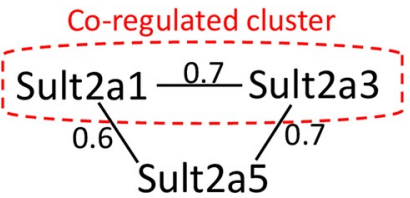

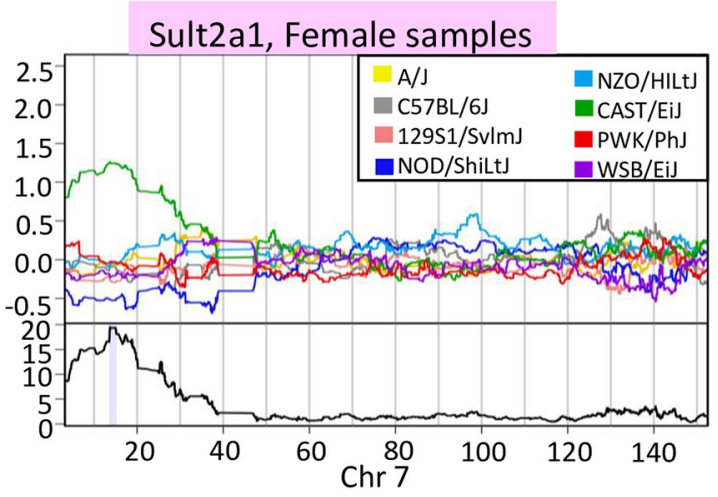

Sult2a3, Female samples

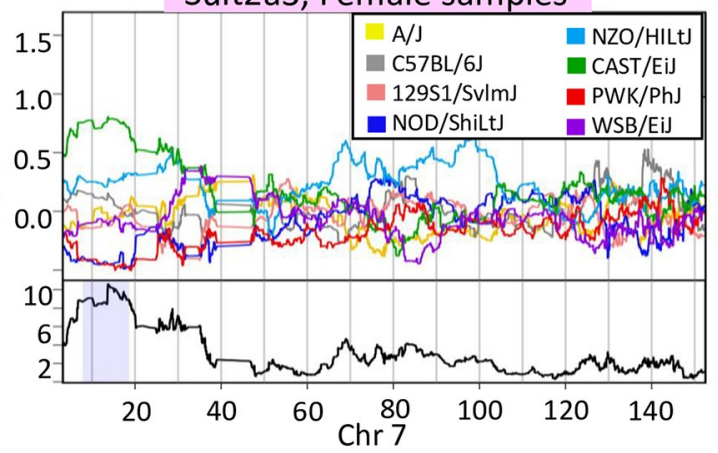

Sult2a5, Female samples

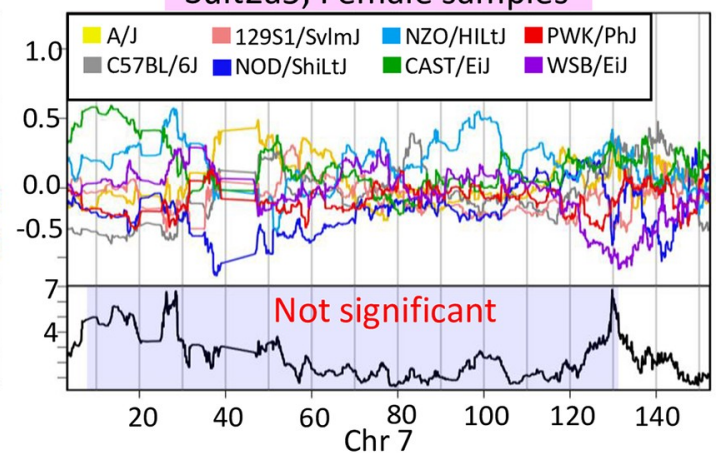

Fig 6. Co-regulated gene clusters that show different gene members in male vs. female liver. (A) Pearson gene expression correlation of Sult2a1, Sult2a3 and Sult2a5 in male liver (left) and female liver (right). (B) Regression coefficients (top figure in each panel) and LOD scores (bottom figure in each panel) across chr7 for Sult2al in male liver (top left) and female liver (top right), for Sult $2 a 3$ in male liver (middle left) and female liver (middle right) and for Sult $2 a 5$ in male liver (bottom left) and female liver (bottom right). Shaded area in the LOD score figures indicate 95\% Bayesian credible interval for each eQTL.

https://doi.org/10.1371/journal.pone.0242665.g006 
Sult2a1 and Sult2a3 show higher correlation in female liver samples, as compared to male liver samples (Pearson correlation 0.7 vs. 0.3 ), and Sult2a1 and Sult2a5 show higher correlation in the male samples, as compared to female samples (Pearson correlation 0.8 vs 0.6) (Fig 6A). The relatively strong correlation in female livers between Sult2a1 and Sult2a5, which was not seen in the CAST/EiJ co-regulated cluster, indicates that there is, nevertheless, an overall correlation in their expression across female DO mouse livers, once the complexity of other DO founder strains is factored in.

\section{Discussion}

Sex-specific gene expression is widespread in mammalian liver, where it impacts male-female differences in lipid and drug metabolism as well as disease susceptibility. While the role of hormonal factors, most notably $\mathrm{GH}$, in regulating liver sex differences is well established, little is known about the effects of genetic factors on the observed differences between the sexes. Here, we used the DO mouse model, a genetically diverse population derived from eight inbred mouse strains [57], to dissect the genetic component of sex-biased transcriptional regulation. We discovered that an unexpectedly large number of distinct genetic factors work in tandem with the hormonal environment to regulate the transcription of sex-biased genes in the liver. DO mice showed high variability in sex-specific gene expression that reflects the high variability of expression seen in the eight founder strains, with $96 \%$ of sex-specific genes deficient in sex-biased expression in one or more strains. We identified 987 associations between autosomal genetic variants and expression of sex-biased genes (eQTLs for sex-biased genes), including 157 eQTLs associated with 130 sex-biased multi-exonic lncRNA genes [20]. Many of these eQTLs showed large differences in genetic association (LOD score) between male and female liver populations and were closely linked to the loss or gain of sex-biased gene expression in the predicted regulating founder mouse strain. Genomic regions that contain genetic variants specific to the regulating founder strain and show sex differences in chromatin accessibility, or in the binding of the GH-regulated transcription factor STAT5, were significantly enriched for eQTLs associated with genes with matching sex-specificity, supporting the proposed functional, regulatory nature of the eQTL regions we identified. Gene clusters defined by overlapping eQTLs identified sets of co-regulated genes, in some cases from different chromosomes, consistent with the trans-acting nature of a subset of the eQTLs described here.

\section{Variability of sex-specific genes across DO founder mice}

We found exceptional high variability of sex-specific gene expression across DO founder mouse strains, with some strains expressing many more sex-specific genes than others, and with sex-specific expression absent in at least one founder strain for nearly all sex-specific genes. This suggests that, for the vast majority of sex-specific genes, conservation of the sexspecificity of a given pathway or function, such as cytochrome P450 (CYP)-mediated lipid and drug metabolism, may be more important than conservation of the sex specificity of individual genes. For example, although sex specificity was preserved across all eight DO founder strains for only 4 of 28 sex-specific Cyp genes (male-specific Cyp2d9 and Cyp4a12a; female-specific Cyp2a4 and Cyp17a1), six of the strains expressed at least 15 sex-specific Cyps each, and the other two strains expressed 9-11 sex-specific Cyp genes each (S1 Table). However, strain-specific enrichment of certain KEGG pathways was also seen, e.g., pyruvate metabolism in WSB/ EiJ mice and biosynthesis of unsaturated fatty acids in $\mathrm{PWK} / \mathrm{PhJ}$ mice. Of the 20 genes that showed consistent sex-specific expression across all eight strains, eight genes were active in steroid and drug/foreign chemical metabolism (Cyp, Gst, Fmo, Aox, and Hsd family members), three were enzymes of fatty acid biosynthesis and metabolism (Elovl3, Acot3, Cyp4a12a), two 
were complement genes (C8b, C9), and two were involved in liver fibrosis (Prom1, Rtn4; [74, 75]). The conserved sex specificity of these 20 genes suggests they carry out essential, nonredundant sex-specific functions. The strain-dependent expression seen for the much larger number of other sex-specific genes is consistent with the diverse hepatic phenotypes, including differences in sex-biased liver disease susceptibility, seen in various mouse strains and in outbred individuals $[57,76]$.

Genetic variants accounted for a large fraction of the variability of sex-biased gene expression seen in DO mouse liver. Thus, $68 \%$ of sex-specific genes examined (i.e., 322 of 471 sexspecific genes for which we have DO founder expression data) were regulated by a set of 360 eQTLs predicted to either decrease or increase sex-specificity in the regulating founder strain. The effects of these eQTLs act by a variety of mechanisms, e.g., by suppression of a male-specific gene or activation of a female-specific gene in male liver, which both decrease sex specificity; and by increasing activation of a male-specific gene or increasing suppression of a femalespecific gene in male liver, both of which increase sex specificity. Remarkably, the genetic modifiers of sex-specific genes that we identified explain more than 200 instances of gain or loss of sex-specificity across the eight DO mouse founder strains, highlighting the widespread impact of genetic regulatory factors on the variability of sex-specific gene expression.

DO founder mice exhibit diverse liver phenotypes, including differences in susceptibility to NAFLD [77], liver fibrosis [78] and hepatotoxicity [79, 80], as well as differences in liver inflammatory responses [81], whose molecular mechanisms, involve, at least in part, sexbiased genes [17, 18, 82-85]. Notably, the sex-dependent eQTLs identified here include cis-acting eQTLs for several sex-specific genes implicated in NAFLD, such as Cyp7b1, a male-specific P450 that inactivates estrogenic and hepatotoxic sterols and is a key driver of NAFLD [86-91], Gstp1, a male-biased oxidative stress-protective glutathione transferase with high risk allelic variants for NAFLD in humans [92-94], and Nox4, a male-biased NADPH oxidase that increases hepatocyte oxidative stress, apoptosis and liver fibrosis [95-97]. We also identified both cis and trans sex-dependent eQTLs for Sult3a1, a female-specific hepatic sulfotransferase that protects from benzene genotoxicity [98]. The fact that a majority of genetic risk factors for complex diseases, including sex-biased NAFLD and insulin resistance [91, 99-101], are in non-coding regions, primarily at active DHS [102-105], supports the proposal that the underlying SNPs/indels for many of the sex-dependent eQTLs that we identified are in DHS and other such regulatory regions.

\section{Relationship between genetic variants and GH regulatory mechanisms}

Many of the eQTLs for sex-specific genes described here were substantially stronger in one sex than the other, indicating the underlying SNPs/indels act in a sex-dependent manner. The underlying mechanisms for these effects likely involve transcriptional and epigenetic regulatory events controlled by GH $[4,34,35]$, the key regulator of sex-specific gene expression in the liver. Global mechanisms of sex-specific hormonal regulation are apparently maintained in the DO founder strains, as indicated by their very similar distributions of sex-specific genes across four distinct sex-specific gene classes, defined by their responses to the loss of GH signaling following hypophysectomy [30, 67]. Moreover, sex-biased open chromatin regions (DHS) and sex-biased, GH-regulated STAT5 binding sites containing strain-specific variants were significantly enriched at eQTL regions regulating correspondingly sex-specific genes, while binding of the male-biased, GH-regulated repressor BCL6 $[32,73]$ was most highly enriched at trans-eQTL regions controlling female-specific genes. These findings support the proposal that SNPs/indels at these GH-dependent regulatory regions cause either a loss or a gain in sex-biased transcription factor binding, and thereby alter GH-regulated gene 

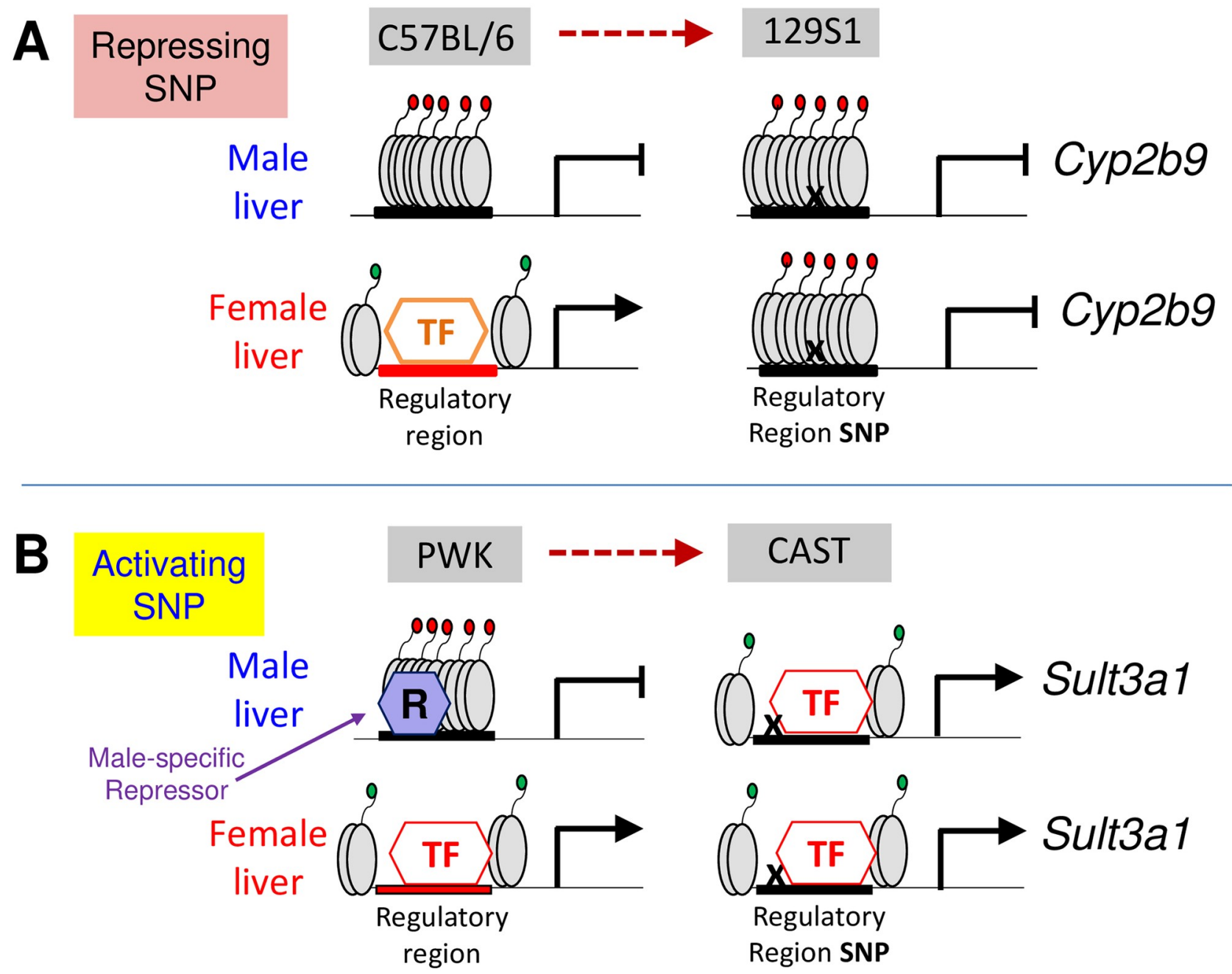

Fig 7. Models for how eQTLs may act in a sex-dependent manner to regulate sex-specific genes. (A) Repressing SNP at $C y p 2 b 9$ regulatory element. A cis-acting eQTL on chr7 with a strong genetic association in female but not male mouse liver represses expression of Cyp2b9 in female 129S1/SvImJ liver (S5D Fig; category \#4 eQTL, Fig 3C). This eQTL is proposed to involve a SNP ('X') that abolishes binding of a female-specific transcriptional activator (TF) to the regulatory element, and may lead to loss of female-biased chromatin accessibility and the loss of the sex-specific expression seen in 129S1/SvImJ liver. The Cyp2b9 eQTL region includes two female-biased DHS, three female-biased STAT5 binding sites, and five female-biased HNF6 binding sites, all of which overlap with 129S1/SvImJ-specific SNPs/indels (S4 Table, S10 Fig). (B) Activating SNP at Sult3a1 regulatory element. A cisacting eQTL on chr10 with a much stronger genetic association in male than female mouse liver enables elevated expression of Sult3a1 in male CAST/ EiJ liver (Fig 2C and 2D; category \#2 eQTL, Fig 3C). This eQTL is proposed to involve a SNP ('X') that abolishes binding of a male-specific repressor protein $(\mathrm{R})$ to the regulatory element shown, but does not interfere with binding of the transcriptional activator (TF), thereby causing loss of the sexspecific expression of Sult3a1 in CAST/EiJ liver (Fig 2E). The chr10 Sult3a1 eQTL region includes two female-biased DHS, one female-biased STAT5 binding site and seven female-biased HNF6 binding sites [31], all of which overlap with 129S1/SvImJ-specific SNPs/indels (S4 Table).

https://doi.org/10.1371/journal.pone.0242665.g007

expression in a sex-dependent manner. In one model (Fig 7A), a strain-specific SNP/indel at one of the sex-biased regulatory elements associated with an eQTL with a strong repressive effect on Cyp2b9 expression in female 129S1/SvImJ liver (S5D Fig) interferes with the binding of factors required for Cyp2b9 transcription. In another model (Fig 7B), an activating SNP/ indel at one of the sex-biased regulatory regions is proposed to explain the de-repression of Sult3a1 in CAST/EiJ male liver (Fig 2C). Further studies, including mutation of individual strain-specific SNPs/indels found at each of the regulatory regions in these eQTLs will be required to fully evaluate these proposed mechanisms. 


\section{Properties of the genetic regulation of sex-biased genes}

Our findings give insight into several important features that govern how genetic modifiers impact the expression of sex-specific genes in the liver. We found major differences in the sexspecific liver transcriptome between mouse strains, determined by an unexpectedly large number of individual genetic loci, each regulating small numbers of genes in cis and/or in trans, and without affecting the overall patterns of GH regulation that dictate sex-specific gene transcription in the liver. Thus, the co-regulated gene clusters we identified from overlapping eQTLs in the same regulating strain are mostly small in size, containing five or fewer genes. However, this finding does not preclude the possibility that some of the clustered genes are themselves regulators of larger numbers of downstream genes, but whose effects are not sufficiently strong to be discovered as eQTLs in our analyses. Genetic modifiers of sex-specific gene expression may also operate via regulatory proteins that are not themselves expressed in a sex-dependent manner. One such example is Rsll, which codes for a Krüppel-associated box (KRAB) zinc finger repressor whose expression varies across mouse strains $[106,107]$ and for which we found both cis and trans eQTLs (S4 Table). Rsl1 directly represses Slp and a few other male-specific genes in mouse liver, but can also regulate other sex-specific genes by indirect activation or repression, which enables genetic variants of $R s l$ to have a more widespread impact on sex-specific gene expression patterns $[42,43]$. Another feature of genetic regulation seen here is the clustering of protein coding genes with co-regulated lncRNA genes, which may contribute to sex-specific gene regulation [20] through epigenetic or other regulatory mechanisms [82, 108, 109]. Finally, our finding of large numbers of sex-biased autosomal genetic associations in mouse liver differs from results in whole blood based on clinical samples, where a common autosomal genetic architecture for gene expression was found across the sexes [110].

\section{Conclusions}

Male-female differences in liver gene expression confer sex differences in many biological processes relevant to health and disease, including lipid and drug metabolism and liver disease susceptibility. While the role of hormonal factors, most notably growth hormone, in regulating hepatic sex differences is well established, little is known about how autosomal genetic factors impact sex differences on an individual basis. Here, we harness the power of mouse genetics provided by the Diversity Outbred mouse model to discover significant genome-wide associations between genetic variants and sex-specific liver gene expression. Remarkably, we found that autosomal expression quantitative trait loci with a strong sex-bias account for the loss or gain of sex-specific expression of more than 200 autosomal genes seen across eight founder mice strains. Genetic associations with sex-specific genes were enriched for sex-biased and growth hormone-dependent regulatory regions harboring strain-specific genetic variants. Coregulated gene clusters identified by overlapping regulatory regions included highly correlated genes from different chromosomes. These findings reveal the extensive regulatory role played by autosomal genetic variants, working in tandem with growth hormone signaling pathways, in the transcriptional control of sex-biased genes, many of which have been implicated in sex differential outcomes in liver metabolism and disease susceptibility.

\section{Materials and methods}

\section{DO mouse liver datasets and analyses}

Liver RNA sequencing expression data was downloaded from GEO (https://www.ncbi.nlm. nih.gov/geo/) for 438 individual DO mice using accession numbers GSE45684 [68, 69] and 
GSE72759 [46]. The datasets used for our analyses were derived from 219 male and 219 female DO mice, 26 weeks of age, all with SNP array data available. Of these, 112 males and 110 females were fed a standard chow diet, and 107 males and 109 females were fed a high fat diet. Genotyping data based on SNP arrays were downloaded from the DO Database (https://www. jax.org/research-and-faculty/genetic-diversity-initiative/tools-data/diversity-outbreddatabase\#) for 264 DO samples genotyped at 7,854 SNPs using the Mouse Universal Genotyping Array (MUGA) [111] and for 174 DO samples genotyped using a higher density array, MegaMUGA [112], encompassing 77,725 SNP probes. Haplotype reconstruction: Quantile normalization, implemented in DOQTL [113], was used to normalize microarray intensity values across batches with default parameters (S3A Fig). DOQTL was applied to the normalized microarray intensity values at each SNP probe to determine the founder strain that the SNP is most likely inherited from, i.e. the founder haplotype, as follows. Two founder haplotypes, referred to as founder diplotype, are possible at each SNP locus, giving a total of 36 possible founder diplotypes for a population generated from 8 founder strains: 8 homozygous diplotypes and 28 heterozygous diplotypes. DOQTL uses the intensity of each SNP probe to generate a probabilistic estimate for each diplotype state at each SNP in each DO mouse using a hidden Markov model, where the hidden states are the diplotype states. DOQTL assigned each SNP locus to the diplotype state with the highest posterior probability. Individual mouse genome reconstruction: Seqnature [69] was used to construct an individual diploid genome for each DO mouse using founder haplotypes that were inferred at the previous step. Recombination boundaries were defined as the genomic midpoint between neighboring SNPs that were assigned to different founder diplotypes. For each recombination block, Seqnature was used to recapitulate the founder genome of interest by incorporating high quality SNPs and small indels ( $<100$ bases) that were found in that particular genome, downloaded as a vcf file from the Sanger Mouse Genome project (release 1211, [63]). RNA-seq read mapping: Tophat2 $[114,115]$ was used with default parameters to map RNA-seq reads from each individual DO mouse liver sample to that mouse's diploid genome, which was comprised of both paternal and maternal allele sequences (S3A Fig).

\section{Other mouse liver RNA-seq samples and data analyses}

Three publicly available liver gene expression datasets were downloaded for the eight DO mouse founder strains: 1) a microarray dataset comprised of 96 samples, six replicates per sex for each strain, assayed on the Illumina Sentrix Mouse-6 V1.1 platform, at http://cgd.jax.org/ gem/strainsurvey26/v1; 2) an RNA-seq dataset comprised of 128 male liver samples, six livers per strain from each of two diets, standard chow and high fat diet (GEO accession GSE45684) $[68,69]$; and 3) an RNA-seq dataset comprised of 12 male and 12 female mouse livers for strain C57Bl/6J (GEO accession GSE59222) [3]. Tophat2 was used with default parameters to map each RNA-seq sample to its respective strain's genome, generated by Seqnature based on SNPs/indels retrieved from the Sanger Mouse Genome project (release 1211) [63]. RNA-seq data for livers from the CD-1 (ICR) mouse strain, consisting of three pools of male and three pools female liver samples, was downloaded from GEO accession GSE98586 [34] and then mapped to the reference mouse genome using Tophat 2 .

\section{Expression quantification}

Gene expression was quantified using featureCounts [116] to count sequence reads that overlap any exon by at least one bp. For RNA-seq datasets that were mapped to a haploid genome, e.g. the $\mathrm{C} 57 \mathrm{Bl} / 6 \mathrm{~J}$ reference mouse genome and the eight $\mathrm{DO}$ mouse founder strains, only uniquely mapping reads were used. For DO mouse datasets, which were mapped to the 
reconstructed diploid genome of each individual mouse, the best mapped location for each read was used. The restriction of using only unique mapped reads was not imposed, as it would exclude reads that map to genomic regions where the paternal and maternal alleles are the same, which in many cases would substantially underestimate expression of a gene. Gene expression levels for DO mouse RNA-seq data were based on the total number of reads that overlap the counted regions in either the paternal or maternal allele. Read counts were then transformed to fragments per kilobase of exon per million reads mapped (FPKM) for downstream analysis. For FPKM calculation on diploid genomes, exon lengths were based on the average length of exons for the two alleles of each gene. See S8 Table for full expression data for each liver.

\section{Sex-specificity of gene expression}

Three liver expression datasets, described above, were used to establish the sex-specificity of protein-coding genes: 1) male and female CD1 mouse liver RNA-seq dataset; 2) male and female C57Bl/6J mouse liver RNA-seq dataset; and 3) male and female mouse liver Illumina Sentrix Mouse-6 V1.1 expression microarray dataset, encompassing each of the eight DO mouse founder strains. edgeR [117] was used to perform differential analysis for the two RNAseq datasets; and limma [118] with default parameters was used for the microarray dataset. For genes with multiple microarray probes, we chose the probe with the lowest adjusted p-value. We considered a total of 1,196 sex-specific genes, as follows. A total of 1,033 protein-coding genes were designated sex-specific (S1 Table) based on the following criteria: male/female gene expression |fold-change| $>2$ at FDR $<0.05$ in at least one RNA-seq dataset, or |foldchange $>1.5$ at FDR $<0.05$ in at least one of the microarray datasets. A set of 168 multiexonic, intergenic liver-expressed lncRNAs designated sex-specific based on their significant sex-biased expression in one or more of the 8 RNA-seq datasets examined previously (male/ female |fold-change| $>4$ at FDR $<0.05$; see listing in S2A Table, column AB, of [20]), of which 163 were expressed in at least one DO liver sample. For each gene, the sex specificity was based on its sex bias in the strain with the largest |fold-change| for male/female expression ratio. Where indicated, our analyses were limited to 471 sex-specific protein-coding genes for which microarray expression data is available for all eight DO founder strains in both sexes.

\section{Variability of gene expression across DO founder strains}

RNA-seq data for $\mathrm{n}=8$ male livers in each DO founder mouse strain was used to determine the variability across, and within, each founder strains, as follows: Inter-strain variability $($ Gene $\mathrm{X})=$ [standard deviation of (mean expression value of Gene $\mathrm{X}$ in each of the 8 strains)] / [average of (set of mean expression values for Gene $\mathrm{X}$ in each of the 8 strains)]. Intra-strain variability $($ Gene $\mathrm{X})=[$ standard deviation of Gene X expression across $\mathrm{n}=8$ male livers in strain $s$ ] / [mean of Gene X expression in $\mathrm{n}=8$ male livers in strain $s$ ].

\section{eQTL analysis}

eQTLs were identified using liver gene expression data 219 male and 219 female DO mouse livers (S3A Fig, S2 Table). We used the additive haplotype model in DOQTL [113] to perform QTL mapping by regressing each gene's expression level based on the estimates of each founder strain's contribution, i.e., the founder diplotype probability, at each SNP marker. Adjustments to account for relatedness amongst DO mice, as well as batch, sex, diet, and interaction between sex and diet were included in the regression analysis as additive covariates, written as the following in R: model.matrix $\left(\sim \operatorname{sex}+\operatorname{diet}+\operatorname{diet}^{*} \operatorname{sex}+\right.$ generation + batch, data $=$ data). The strength of the association between gene expression and genotype is given as 
a likelihood ratio (LOD score, logarithm of the odds score), which is the -log10(p-value) when comparing the full model to the null model, where the null model excludes diplotype probabilities. QTL mapping also gives eight regression coefficients, whose magnitudes reflect the effect of the founder alleles at each SNP marker. A positive regression coefficient indicates the genetic variant is associated with high expression of the gene of interest, and a negative regression coefficient indicates the genetic variant is associated with low expression of the gene of interest. For each eQTL, the regulating strain (i.e., the strain with the largest alteration in gene expression due to genetic factors) was defined as the DO founder strain whose regression coefficient had the largest absolute value. A Bayesian credible interval, defined as $95 \%$ of the region under the LOD^ 10 curve, as implemented in DOQTL [113], was defined for each eQTL. This interval delineates the genomic location where the highest association occurs, as defined by the uppermost $5 \%$ area under the peak of the LOD score curve. Significance level: A genomewide p-value for each association was determined by carrying out 1,000 permutations on the gene expression data. To assemble a list of associations between gene expression and SNPs, the SNP with the highest LOD score for each gene was recorded; subsequent high-scoring SNPs were retained if their $\mathrm{p}$-value was $<0.05$ and if they were located on a different chromosome than the SNP with the highest LOD score. An FDR correction was then applied to the genome-wide p-values. Any eQTL with FDR $<0.05$ was deemed significant. To identify genetic variants whose association with gene expression was only found in one sex, or was stronger in one sex than in the other, we repeated the eQTL mapping analysis twice more, once using only the 219 male DO liver samples, and a second time using only the 219 female DO liver samples. Gene expression pre-processing: Gene expression levels, in units of FPKM, were transformed into normal scores using the inverse normal transformation in DOQTL [113] prior to their use for eQTL mapping. eQTL mapping was carried out for protein-coding genes and for intergenic, multi-exonic lncRNA genes that were expressed in at least one liver sample, namely: 20,559 genes (18,543 protein-coding genes and multi-exonic 2,016 lncRNA genes) when analyzing the 438 male and female DO livers together as a single set; 20,268 genes (18,275 protein-coding and 1,993 lncRNA genes) when analyzing only the 219 male DO livers; and 20,190 genes (18,207 protein-coding and 1,983 lncRNA genes) when analyzing only the 219 female DO livers. Interpolating missing genotype information: To preserve the extra information that the higher density SNP array provides, QTL mapping was performed on 64,713 SNPs markers (S2A Table), which corresponds to the union of SNP markers from the two genotyping arrays used after removal of SNP probes with no differentiating information across the eight strains [112]. For missing SNP probes, i.e. SNPs that were unique to one type of array, diplotype probabilities were assigned from the nearest measured SNP. All of the SNP probes that were analyzed are located on autosomes or on chrX. Novelty of eQTLs: An eQTL was considered novel if its $95 \%$ Bayesian credible interval did not overlap by at least one bp the associated variant region of a published eQTL for the same gene name or RefSeq accession number. Cis and trans eQTLs: An eQTL was designated cis if the eQTL's 95\% Bayesian credible interval overlapped (by at least $1 \mathrm{bp}$ ) the TAD region that contains the transcription start site of the gene regulated by the eQTL; otherwise it was designated a trans eQTL. TAD coordinates were those listed for mouse liver in [72].

\section{Categorization of eQTLs with a sex-bias in genetic association}

Sex-biased genetic associations were defined for 360 autosomal eQTLs that are significant in either male liver or female liver samples (see S4A Table, columns D, F, N and O), based on LOD score calculated using male only DO liver samples minus LOD score calculated using female only DO liver samples. Each such sex-dependent eQTL was assigned to one of eight 
categories based on three criteria: the sex-specificity of the gene it is associated with, the sexbias of its genetic association (LOD score in males minus LOD score in females), and its regression coefficient in the regulating strain and in the sex where the eQTL LOD score is higher ( 55 Table). A decrease (or an increase) in sex specificity was inferred from a negative (or a positive) regression coefficient, which indicates that the gene is predicted to be expressed at a lower (or higher) level in the regulating strain as compared to its expression in the other DO founder strains based on the regression model. For example: category \#1 is comprised of eQTLs associated with male-specific genes that show stronger regulation in male liver (LOD score in males $>$ LOD score in females) and a negative regression coefficient in the regulating strain, indicating a decrease in sex specificity compared to what it would have been without the eQTL; while category \#8 is comprised of eQTLs associated with female-specific genes that show stronger regulation in female liver (LOD score in females $>$ LOD score in males) and a positive regression coefficient in the regulating strain, indicating an increase in sex specificity compared to what it would have been without the eQTL (S5 Table).

\section{Transcription factor binding sites and DHS (open chromatin regions) with strain-specific SNPs/indels at eQTLs}

High quality SNPs/indels in the eight DO mouse founder strains were downloaded from the Sanger Mouse Genome project (release 1211) [63]. SNPs/indels that occur in only one founder strain were designated strain-specific variants, and were extracted from a variant call format (vcf) file downloaded from https://www.sanger.ac.uk/sanger/Mouse_SnpViewer/rel-1211 (release 1211) using a custom R-script provided in S1 File. We selected for analysis DHS and transcription factor binding site ChIP-seq peaks that are within each eQTL region (95\% Bayesian Credible interval, based on the liver sample set that gives the highest LOD score), and whose peak region contains a strain-specific $\mathrm{SNP} /$ indel for the matching regulating strain. For these analyses, we considered eQTL regions that are $<3 \mathrm{MB}$ in width $(8,254$ of all 12,886 autosomal eQTLs). Further, eQTLs identified for genes with |male/female| expression $>4$ were designated an eQTL for a sex-specific gene, and eQTLs identified for genes with |male/ female $\mid<1.2$ were designated an eQTL for a sex-independent gene, based on male/female expression ratios determined by edgeR. The number of eQTLs included in the analysis of DHS and transcription factor binding sites ranged from 17 and 2,793, and is specified for each analysis in the x-axis labels of Fig 4. For each eQTL region, we counted the fraction of DHS or ChIP-seq binding sites for each transcription factor analyzed that are male-specific, femalespecific or sex-independent, as defined in the published studies for STAT5 [32] and BCL6 (see S11 Table of [21]), and for the set of 72,000 liver DHS from [33], of which 4,644 DHS were designated male-biased DHS and 2,814 were designated female-biased DHS based on reanalysis of the published raw data performed by Gracia Bonila of this laboratory. For each eQTL considered, we computed the fraction of all male-specific, female-specific and sex-independent DHS, or transcription factor binding sites, within the eQTL region that contain a SNP/indel specific to the regulated strain for that eQTL (S6 Fig), and then compared the distributions of those fractions to those of the other sets of eQTLs being considered, as shown along the $\mathrm{x}$-axis of Fig 4. Significance was determined by Wilcoxon test.

\section{Co-regulated gene clusters}

Each eQTL region was centered at the SNP marker with the highest LOD score, i.e. the eQTL peak. eQTL region boundaries were set at the second SNP marker from the center; thus, each eQTL region contains five SNP markers. eQTL regions with the same regulating strain whose boundaries overlap by at least one bp were assigned to the same co-regulated gene cluster. The 
genomic region encompassing overlapping eQTLs was computed two ways: 1) based on the intersection of the regions defined by the five SNP markers of each eQTL; and 2) based on the union of the $95 \%$ percent Bayesian intervals of each of the overlapping eQTLs. Of note, the 95\% percent Bayesian interval may be smaller or larger than the five SNP region, and the union of $95 \%$ Bayesian intervals may also be smaller than the intersection of the five SNP regions, depending on how large and overlapping the 95\% Bayesian intervals are. Co-regulated gene clusters were identified based on eQTLs discovered in all DO liver samples, and separately, male only DO liver samples, and female only DO liver samples. This analysis considered eQTLs for genes that are expressed (FPKM $>0$ ) in at least $25 \%$ of each set of liver samples, i.e., all DO livers, male only DO livers, or female only DO livers.

\section{Other analyses}

The var function in $\mathrm{R}$ was used to quantify liver expression variation for sex-specific genes across livers from 112 chow diet-fed male DO mice, and separately across livers of 110 chow diet-fed female DO mice. Sex-specific genes belonging to each of four classes, defined by their responses to hypophysectomy, were from S3 Table of [30]. The ComBat function in the sva $\mathrm{R}$ package [119] was used with default parameters to remove from the gene expression data (in units of $\log 2(\mathrm{FPKM}+1))$ a batch effect that correlated with the liver sample's GEO accession number. Pearson correlation was then used to calculate pairwise gene expression correlations. PCA was implemented using the prcomp function in R using log2, centered normalized microarray intensity values. DAVID functional annotation (https://david.ncifcrf.gov/) [65, 66] was used to discover enriched KEGG pathways by inputting the official gene symbols for the sex-specific protein-coding genes discovered in each strain. Pathways with adjusted P (Benjamini) $<0.05$ were deemed significant. liftOver [120] was used to convert genomic regions into mouse $\mathrm{mm} 9$ coordinates using default parameters, and bioDBnet:db2db [121] was used to convert Ensembl gene identifiers to RefSeq accession numbers.

\section{Supporting information}

S1 Fig. High variability of the sex-specificity of gene expression in individual livers of CD1 mice and DO mouse founder strains for two examples of sex-specific genes. Gene expression in individual mouse livers across strains: male and female CD-1 mice (first row; left), male and female C57BL/6J mice (first row, right), male and female DO mice fed a standard chow diet (second row), or fed a high fat diet (third row). The fourth row shows box plots of gene expression level (FPKM) based on 128 individual male livers for DO founder mice fed a standard chow diet (left), or fed a high fat diet (right), and for male and female DO founder strain mice (fifth row; left). The first four rows present gene expression values determined by RNAseq (FPKM values), while the gene expression on the fifth row was determined by microarray analysis. Male/Female expression ratios across the DO founder strains based on the microarray dataset are also presented (fifth row; right). Examples shown are for Cyp4a12b, a male-specific gene (A), where male-biased expression is reduced or lost in $\mathrm{PWK} / \mathrm{PhJ}$ mice, and for Cyp2c39, a female-specific gene (B), where female-biased expression is lost in C57BL/6J mice. (PPTX)

S2 Fig. Numbers of sex-specific protein-coding genes in each DO founder strain. Shown are the number of sex-specific protein-coding genes (A) or number of highly sex-specific protein-coding genes $(\mathbf{B})$ (male/female $\mid$ fold-change $\mid>4$ at FDR $<0.05$ ) in at least one DO mouse founder strain based on the microarray dataset) for each founder mouse strain. (PPTX) 
S3 Fig. Analysis of SNP array and RNA-seq data for each DO mouse liver sample. Schematic overview (A), and properties of autosomal eQTLs in DO mouse liver (B). Shown in (B) are the percentages of autosomal eQTLs that are associated with increased expression (white bars) or decreased expression (colored bars) in the respective regulating founder mouse strain. These data are based on $625,672,574,593,616,3,085,3,074$ and 1,086 autosomal eQTLs whose regulating strain is A/J, C57BL/6J, 129S1/SvlmJ, NOD/ShiLtJ, NZO/HILtJ, CAST/EiJ, $\mathrm{PWK} / \mathrm{PhJ}$, or WSB/EiJ, respectively. Data are based on the 10,325 autosomal eQTLs (Table 1) discovered when the set of all DO liver samples were used for eQTL discovery. (PPTX)

S4 Fig. Examples of sex-specific genes with stronger genetic association in male or female liver. For each gene, the figure shows the gene expression patterns across strains (see S1 Fig for details), and genome-wide eQTL scans using the three different DO liver datasets, where the horizontal red line marks the $\mathrm{P}<0.05$ significance cutoff for eQTL significance based on the permutation test. Also shown are regression coefficients across the chromosome that contains the significant eQTL peak, as follows: Regression coefficients (top of each panel) and LOD scores ( $\log 10$ (p-value); bottom of each panel) across the chromosome that has a significant eQTL peak in male (left) or female (right) mouse liver, as marked at bottom. Shaded area in the LOD score plot indicates the 95\% Bayesian credible interval for each eQTL. (A) Cav1 shows strong male-specific expression in the $\mathrm{PWK} / \mathrm{PhJ}$ founder strain and in a subset of male DO mouse livers. Its strong eQTL is seen in male livers only and is associated with the PWK/ $\mathrm{PhJ}$ strain. (B) Hsd3b5 shows male-biased expression in CD-1 mice and multiple DO founder strains; its expression is repressed in female C57BL/6J mice. (PPTX)

S5 Fig. Examples of sex-specific genes in categories \# 1-8, described in Fig 3. For each gene, the figure shows gene expression patterns across strains, genome-wide eQTL scan, and regression coefficients at the chromosome with a significant peak, as described in the legend to S1 Fig. (PPTX)

S6 Fig. Enrichment of sex-specific regulatory elements with strain-specific SNPs/indels at eQTLs for sex-specific genes. In the example shown, 5 of the 8 regulatory elements (i.e., ChIP-seq binding sites) within the eQTL region shown are male-specific binding sites, three of which contain either SNPs or Indels specific for the regulating strain. A fourth regulatory element with a strain-specific SNP is a sex-independent binding site. One strain-specific SNP is not in a regulatory element, and so is excluded from the analyses shown in Fig 4, as are the four reguatory elements without any strain-specific SNPs/indels. (PPTX)

S7 Fig. Regression coefficients (top figure in each panel) and LOD scores (bottom figure in each panel) for genes shown in Fig 5A. (PPTX)

S8 Fig. Genome-wide LOD score plots for genes that are located in different chromosomes in the co-regulated cluster depicted in Fig 5A. (PPTX)

S9 Fig. Genome-wide LOD score plots the Sult2a1, Sult2a3 and Sult2a5 genes. (PPTX)

S10 Fig. Strain-specific SNPs/indels proposed to contribute to the loss of sex-specific gene expression for $C y p 2 b 9$ in female $129 S 1 / S v l m J$ liver. (A) distribution of gene expression level 
of Cyp2b9 across individual DO mouse livers (S2 Table) was used to discover eQTLs stratified by the genotype assigned at the the SNP marker with the highest LOD score. (B) A femalebiased STAT5 binding sites (fourth red arrow from the top) at a female-biased DHS (third red arrow from the top), containing two 129S1/SvlmJ-specific SNPs/indels (two top arrows), located within the eQTL region for Cyp2b9. Although the 129S1/SvlmJ-specific SNPs/indels in the DHS/STAT5 region (top two red arrows) are closer to Cyp2a4 than Cyp2b9, they are in the same TAD region as Cyp2b9, and more importantly, the eQTL affects the expression of Cyp269, but not Cyp2a4. These two genes show a weak negative correlation of their expression in female liver (Pearson correlation $=-0.1$ ) and only a moderate correlation in male liver (Pearson correlation $=0.3)$.

(PPTX)

S1 File. Files for custom R-script used to extract strain-specific SNPs/indels from variant call format file downloaded from https://www.sanger.ac.uk/sanger/Mouse_SnpViewer/rel1211 (release 1211).

(TXT)

S1 Table.

(XLSX)

S2 Table.

S3 Table.

(XLSX)

S4 Table.

(XLSX)

S5 Table.

(XLSX)

S6 Table.

(XLSX)

S7 Table.

(XLSX)

S8 Table.

(ZIP)

\section{Acknowledgments}

We thank Gracia Bonilla of this laboratory for providing lists of male-biased and female-biased DHS used in Fig 4, and Dr. Daniel Gatti of The Jackson Laboratory for providing access to the DO mouse SNP array and liver expression datasets used in this study.

\section{Author Contributions}

Conceptualization: Tisha Melia, David J. Waxman.

Data curation: Tisha Melia.

Formal analysis: Tisha Melia.

Funding acquisition: David J. Waxman. 
Investigation: Tisha Melia, David J. Waxman.

Methodology: Tisha Melia.

Project administration: David J. Waxman.

Software: Tisha Melia.

Supervision: David J. Waxman.

Visualization: Tisha Melia.

Writing - original draft: Tisha Melia, David J. Waxman.

Writing - review \& editing: David J. Waxman.

\section{References}

1. Rinn JL, Snyder M. Sexual dimorphism in mammalian gene expression. Trends in genetics: TIG. 2005; 21(5):298-305. Epub 2005/04/27. https://doi.org/10.1016/j.tig.2005.03.005 PMID: 15851067.

2. Clodfelter KH, Holloway MG, Hodor P, Park SH, Ray WJ, Waxman DJ. Sex-dependent liver gene expression is extensive and largely dependent upon signal transducer and activator of transcription $5 b$ (STAT5b): STAT5b-dependent activation of male genes and repression of female genes revealed by microarray analysis. Molecular endocrinology (Baltimore, Md). 2006; 20(6):1333-51. Epub 2006/02/ 14. https://doi.org/10.1210/me.2005-0489 PMID: 16469768.

3. Lowe R, Gemma C, Rakyan VK, Holland ML. Sexually dimorphic gene expression emerges with embryonic genome activation and is dynamic throughout development. BMC genomics. 2015; 16:295 Epub 2015/04/19. https://doi.org/10.1186/s12864-015-1506-4 PMID: 25888192

4. Lau-Corona D, Bae WK, Hennighausen L, Waxman DJ. Sex-biased genetic programs in liver metabolism and liver fibrosis are controlled by EZH1 and EZH2. PLoS genetics. 2020; 16(5):e1008796. Epub 2020/05/20. https://doi.org/10.1371/journal.pgen.1008796 PMID: 32428001

5. Wauthier V, Waxman DJ. Sex-specific early growth hormone response genes in rat liver. Molecular endocrinology (Baltimore, Md). 2008; 22(8):1962-74. Epub 2008/05/17. https://doi.org/10.1210/me. 2007-0549 PMID: 18483176

6. Yu Y, Fuscoe JC, Zhao C, Guo C, Jia M, Qing T, et al. A rat RNA-Seq transcriptomic BodyMap across 11 organs and 4 developmental stages. Nature communications. 2014; 5:3230. Epub 2014/02/11. https://doi.org/10.1038/ncomms4230 PMID: 24510058

7. Zhang Y, Klein K, Sugathan A, Nassery N, Dombkowski A, Zanger UM, et al. Transcriptional profiling of human liver identifies sex-biased genes associated with polygenic dyslipidemia and coronary artery disease. PloS one. 2011; 6(8):e23506. Epub 2011/08/23. https://doi.org/10.1371/journal.pone. 0023506 PMID: 21858147

8. Lamba V, Jia B, Liang F. STAT5A and STAT5B have opposite correlations with drug response gene expression. Biochemical and biophysical research communications. 2016; 479(2):117-24. Epub 2016/06/07. https://doi.org/10.1016/j.bbrc.2016.06.011 PMID: 27264955

9. Zanger UM, Schwab M. Cytochrome P450 enzymes in drug metabolism: regulation of gene expression, enzyme activities, and impact of genetic variation. Pharmacol Ther. 2013; 138(1):103-41. https://doi.org/10.1016/j.pharmthera.2012.12.007 PMID: 23333322.

10. Lopes-Ramos CM, Kuijjer ML, Ogino S, Fuchs CS, DeMeo DL, Glass K, et al. Gene Regulatory Network Analysis Identifies Sex-Linked Differences in Colon Cancer Drug Metabolism. Cancer research 2018; 78(19):5538-47. Epub 2018/10/03. https://doi.org/10.1158/0008-5472.CAN-18-0454 PMID: 30275053

11. Waxman DJ, Holloway MG. Sex differences in the expression of hepatic drug metabolizing enzymes. Mol Pharmacol. 2009; 76(2):215-28. Epub 2009/06/02. https://doi.org/10.1124/mol.109.056705 PMID: 19483103

12. Liu W, Kulkarni K, Hu M. Gender-dependent differences in uridine 5'-diphospho-glucuronosyltransferase have implications in metabolism and clearance of xenobiotics. Expert Opin Drug Metab Toxicol. 2013; 9(12):1555-69. https://doi.org/10.1517/17425255.2013.829040 PMID: 24011176.

13. Alnouti $Y$, Klaassen $C D$. Mechanisms of gender-specific regulation of mouse sulfotransferases (Sults). Xenobiotica; the fate of foreign compounds in biological systems. 2011; 41(3):187-97. Epub 2010/11/26. https://doi.org/10.3109/00498254.2010.535923 PMID: 21091322 
14. Wang X, Magkos F, Mittendorfer B. Sex differences in lipid and lipoprotein metabolism: it's not just about sex hormones. J Clin Endocrinol Metab. 2011; 96(4):885-93. Epub 2011/04/09. https://doi.org/ 10.1210/jc.2010-2061 PMID: 21474685

15. Grossmann $M$, Wierman ME, Angus $P$, Handelsman DJ. Reproductive Endocrinology of Nonalcoholic Fatty Liver Disease. Endocrine reviews. 2019; 40(2):417-46. Epub 2018/12/01. https://doi.org/10. 1210/er.2018-00158 PMID: 30500887.

16. Biswas S, Ghose S. Divergent impact of gender in advancement of liver injuries, diseases, and carcinogenesis. Frontiers in bioscience (Scholar edition). 2018; 10:65-100. Epub 2017/09/21. https://doi. org/10.2741/s501 PMID: 28930519.

17. Tessari $P$, Coracina $A$, Cosma $A$, Tiengo $A$. Hepatic lipid metabolism and non-alcoholic fatty liver disease. Nutr Metab Cardiovasc Dis. 2009; 19(4):291-302. https://doi.org/10.1016/j.numecd.2008.12. 015 PMID: 19359149.

18. Baik $\mathrm{M}, \mathrm{Yu} \mathrm{JH}$, Hennighausen $\mathrm{L}$. Growth hormone-STAT5 regulation of growth, hepatocellular carcinoma, and liver metabolism. Ann N Y Acad Sci. 2011; 1229:29-37. Epub 2011/07/29. https://doi.org/ 10.1111/j.1749-6632.2011.06100.x PMID: 21793836

19. Conforto TL, Waxman DJ. Sex-specific mouse liver gene expression: genome-wide analysis of developmental changes from pre-pubertal period to young adulthood. Biology of sex differences. 2012; 3:9. Epub 2012/04/06. https://doi.org/10.1186/2042-6410-3-9 PMID: 22475005

20. Melia T, Waxman DJ. Sex-Biased IncRNAs Inversely Correlate With Sex-Opposite Gene Coexpression Networks in Diversity Outbred Mouse Liver. Endocrinology. 2019; 160(5):989-1007. Epub 2019/ 03/07. https://doi.org/10.1210/en.2018-00949 PMID: 30840070

21. Melia T, Hao P, Yilmaz F, Waxman DJ. Hepatic Long Intergenic Noncoding RNAs: High Promoter Conservation and Dynamic, Sex-Dependent Transcriptional Regulation by Growth Hormone. Mol Cell Biol. 2016; 36(1):50-69. Epub 2015/10/16. https://doi.org/10.1128/MCB.00861-15 PMID: 26459762

22. Hao P, Waxman DJ. Functional Roles of Sex-Biased, Growth Hormone-Regulated MicroRNAs miR1948 and miR-802 in Young Adult Mouse Liver. Endocrinology. 2018; 159(3):1377-92. Epub 2018/01/ 19. https://doi.org/10.1210/en.2017-03109 PMID: 29346554

23. Kwekel JC, Vijay V, Han T, Moland CL, Desai VG, Fuscoe JC. Sex and age differences in the expression of liver microRNAs during the life span of F344 rats. Biology of sex differences. 2017; 8:6. Epub 2017/02/09. https://doi.org/10.1186/s13293-017-0127-9 PMID: 28174625

24. Warnefors M, Mossinger K, Halbert J, Studer T, VandeBerg JL, Lindgren I, et al. Sex-biased microRNA expression in mammals and birds reveals underlying regulatory mechanisms and a role in dosage compensation. Genome research. 2017; 27(12):1961-73. Epub 2017/10/29. https://doi.org/10. 1101/gr.225391.117 PMID: 29079676

25. Waxman DJ, O'Connor C. Growth hormone regulation of sex-dependent liver gene expression. Molecular endocrinology (Baltimore, Md). 2006; 20(11):2613-29. Epub 2006/03/18. https://doi.org/10.1210/ me.2006-0007 PMID: 16543404.

26. Lichanska AM, Waters MJ. How growth hormone controls growth, obesity and sexual dimorphism. Trends in genetics: TIG. 2008; 24(1):41-7. Epub 2007/12/08. https://doi.org/10.1016/j.tig.2007.10.006 PMID: 18063438.

27. Veldhuis JD, Anderson SM, Shah N, Bray M, Vick T, Gentili A, et al. Neurophysiological regulation and target-tissue impact of the pulsatile mode of growth hormone secretion in the human. Growth Horm IGF Res. 2001; 11 Suppl A:S25-37. https://doi.org/10.1016/s1096-6374(01)80005-8 PMID: 11527085.

28. Veldhuis JD, Bowers CY. Human GH pulsatility: an ensemble property regulated by age and gender. Journal of endocrinological investigation. 2003; 26(9):799-813. https://doi.org/10.1007/BF03345229 PMID: 14964431.

29. Shapiro BH, Agrawal AK, Pampori NA. Gender differences in drug metabolism regulated by growth hormone. The international journal of biochemistry \& cell biology. 1995; 27(1):9-20. Epub 1995/01/01. https://doi.org/10.1016/1357-2725(94)00056-5 PMID: 7757886.

30. Connerney J, Lau-Corona D, Rampersaud A, Waxman DJ. Activation of Male Liver Chromatin Accessibility and STAT5-Dependent Gene Transcription by Plasma Growth Hormone Pulses. Endocrinology. 2017; 158(5):1386-405. https://doi.org/10.1210/en.2017-00060 PMID: 28323953

31. Conforto TL, Steinhardt GFt, Waxman DJ. Cross Talk Between GH-Regulated Transcription Factors HNF6 and CUX2 in Adult Mouse Liver. Molecular endocrinology (Baltimore, Md). 2015; 29(9):1286302. https://doi.org/10.1210/me.2015-1028 PMID: 26218442

32. Zhang Y, Laz EV, Waxman DJ. Dynamic, sex-differential STAT5 and BCL6 6 binding to sex-biased, growth hormone-regulated genes in adult mouse liver. Mol Cell Biol. 2012; 32(4):880-96. Epub 2011/ 12/14. https://doi.org/10.1128/MCB.06312-11 PMID: 22158971 
33. Ling G, Sugathan A, Mazor T, Fraenkel E, Waxman DJ. Unbiased, genome-wide in vivo mapping of transcriptional regulatory elements reveals sex differences in chromatin structure associated with sexspecific liver gene expression. Mol Cell Biol. 2010; 30(23):5531-44. Epub 2010/09/30. https://doi.org/ 10.1128/MCB.00601-10 PMID: 20876297

34. Lau-Corona D, Suvorov A, Waxman DJ. Feminization of Male Mouse Liver by Persistent Growth Hormone Stimulation: Activation of Sex-Biased Transcriptional Networks and Dynamic Changes in Chromatin States. Mol Cell Biol. 2017; 37(19). Epub 2017/07/12. https://doi.org/10.1128/MCB.00301-17 PMID: 28694329

35. Sugathan A, Waxman DJ. Genome-wide analysis of chromatin states reveals distinct mechanisms of sex-dependent gene regulation in male and female mouse liver. Mol Cell Biol. 2013; 33(18):3594-610. Epub 2013/07/10. https://doi.org/10.1128/MCB.00280-13 PMID: 23836885

36. Thomson JP, Ottaviano R, Buesen R, Moggs JG, Schwarz M, Meehan RR. Defining baseline epigenetic landscapes in the rat liver. Epigenomics. 2017; 9(12):1503-27. Epub 2017/11/14. https://doi.org/ 10.2217/epi-2017-0029 PMID: 29130343

37. Reizel Y, Spiro A, Sabag O, Skversky Y, Hecht M, Keshet I, et al. Gender-specific postnatal demethylation and establishment of epigenetic memory. Genes \& development. 2015; 29(9):923-33. Epub 2015/05/03. https://doi.org/10.1101/gad.259309.115 PMID: 25934504

38. Garcia-Calzon S, Perfilyev A, de Mello VD, Pihlajamaki J, Ling C. Sex Differences in the Methylome and Transcriptome of the Human Liver and Circulating HDL-Cholesterol Levels. J Clin Endocrinol Metab. 2018; 103(12):4395-408. Epub 2018/05/31. https://doi.org/10.1210/jc.2018-00423 PMID: 29846646

39. Takasugi M, Hayakawa K, Arai D, Shiota K. Age- and sex-dependent DNA hypomethylation controlled by growth hormone in mouse liver. Mechanisms of ageing and development. 2013; 134(7-8):331-7. Epub 2013/05/28. https://doi.org/10.1016/j.mad.2013.05.003 PMID: 23707638.

40. Matthews BJ, Waxman DJ. Impact of 3D genome organization, guided by cohesin and CTCF looping, on sex-biased chromatin interactions and gene expression in mouse liver. Epigenetics \& chromatin. 2020; 13(1):30. Epub 2020/07/19. https://doi.org/10.1186/s13072-020-00350-y PMID: 32680543

41. Sueyoshi T, Kobayashi R, Nishio K, Aida K, Moore R, Wada T, et al. A nuclear factor (NF2d9) that binds to the male-specific P450 (Cyp 2d-9) gene in mouse liver. Mol Cell Biol. 1995; 15(8):4158-66. Epub 1995/08/01. https://doi.org/10.1128/mcb.15.8.4158 PMID: 7623810

42. Krebs CJ, Khan S, MacDonald JW, Sorenson M, Robins DM. Regulator of sex-limitation KRAB zinc finger proteins modulate sex-dependent and -independent liver metabolism. Physiol Genomics. 2009; 38(1):16-28. Epub 2009/04/09. https://doi.org/10.1152/physiolgenomics.90391.2008 PMID: 19351907

43. Krebs CJ, Schultz DC, Robins DM. The KRAB zinc finger protein RSL1 regulates sex- and tissue-specific promoter methylation and dynamic hormone-responsive chromatin configuration. Mol Cell Biol. 2012; 32(18):3732-42. Epub 2012/07/18. https://doi.org/10.1128/MCB.00615-12 PMID: 22801370

44. Hines RN, Koukouritaki SB, Poch MT, Stephens MC. Regulatory polymorphisms and their contribution to interindividual differences in the expression of enzymes influencing drug and toxicant disposition. Drug metabolism reviews. 2008; 40(2):263-301. Epub 2008/05/09. https://doi.org/10.1080/ 03602530801952682 PMID: 18464046.

45. Mugford CA, Kedderis GL. Sex-dependent metabolism of xenobiotics. Drug metabolism reviews. 1998; 30(3):441-98. https://doi.org/10.3109/03602539808996322 PMID: 9710703.

46. Chick JM, Munger SC, Simecek P, Huttlin EL, Choi K, Gatti DM, et al. Defining the consequences of genetic variation on a proteome-wide scale. Nature. 2016; 534(7608):500-5. Epub 2016/06/17. https://doi.org/10.1038/nature18270 PMID: 27309819

47. Gatti D, Maki A, Chesler EJ, Kirova R, Kosyk O, Lu L, et al. Genome-level analysis of genetic regulation of liver gene expression networks. Hepatology (Baltimore, Md). 2007; 46(2):548-57. Epub 2007/ 06/02. https://doi.org/10.1002/hep.21682 PMID: 17542012

48. Gatti DM, Simecek P, Somes L, Jeffery CT, Vincent MJ, Choi K, et al. The Effects of Sex and Diet on Physiology and Liver Gene Expression in Diversity Outbred Mice. bioRxiv. 2017.

49. Gatti DM, Zhao N, Chesler EJ, Bradford BU, Shabalin AA, Yordanova R, et al. Sex-specific gene expression in the BXD mouse liver. Physiol Genomics. 2010; 42(3):456-68. Epub 2010/06/17. https:// doi.org/10.1152/physiolgenomics.00110.2009 PMID: 20551147

50. Tyler AL, Ji B, Gatti DM, Munger SC, Churchill GA, Svenson KL, et al. Epistatic Networks Jointly Influence Phenotypes Related to Metabolic Disease and Gene Expression in Diversity Outbred Mice. Genetics. 2017; 206(2):621-39. Epub 2017/06/09. https://doi.org/10.1534/genetics.116.198051 PMID: 28592500

51. Kur P, Kolasa-Wołosiuk A, Misiakiewicz-Has K, Wiszniewska B. Sex Hormone-Dependent Physiology and Diseases of Liver. International journal of environmental research and public health. 2020; 17(8). Epub 2020/04/16. https://doi.org/10.3390/ijerph17082620 PMID: 32290381 
52. Kurt Z, Barrere-Cain R, LaGuardia J, Mehrabian M, Pan C, Hui ST, et al. Tissue-specific pathways and networks underlying sexual dimorphism in non-alcoholic fatty liver disease. Biology of sex differences. 2018; 9(1):46. Epub 2018/10/23. https://doi.org/10.1186/s13293-018-0205-7 PMID: 30343673

53. Natri HM, Wilson MA, Buetow KH. Distinct molecular etiologies of male and female hepatocellular carcinoma. BMC cancer. 2019; 19(1):951. Epub 2019/10/17. https://doi.org/10.1186/s12885-019-6167-2 PMID: 31615477

54. Wu Y, Yao N, Feng Y, Tian Z, Yang Y, Zhao Y. Identification and characterization of sexual dimorphism-linked gene expression profile in hepatocellular carcinoma. Oncology reports. 2019; 42(3):93752. Epub 2019/07/20. https://doi.org/10.3892/or.2019.7217 PMID: 31322260

55. Meda C, Barone M, Mitro N, Lolli F, Pedretti S, Caruso D, et al. Hepatic ERa accounts for sex differences in the ability to cope with an excess of dietary lipids. Molecular metabolism. 2020; 32:97-108. Epub 2020/02/08. https://doi.org/10.1016/j.molmet.2019.12.009 PMID: 32029233

56. Rankinen T, Sarzynski MA, Ghosh S, Bouchard C. Are there genetic paths common to obesity, cardiovascular disease outcomes, and cardiovascular risk factors? Circulation research. 2015; 116(5):90922. Epub 2015/02/28. https://doi.org/10.1161/CIRCRESAHA.116.302888 PMID: 25722444

57. Churchill GA, Gatti DM, Munger SC, Svenson KL. The Diversity Outbred mouse population. Mammalian genome: official journal of the International Mammalian Genome Society. 2012; 23(9-10):713-8. Epub 2012/08/16. https://doi.org/10.1007/s00335-012-9414-2 PMID: 22892839

58. Schmidt CW. Diversity outbred: a new generation of mouse model. Environ Health Perspect. 2015; 123(3):A64-7. https://doi.org/10.1289/ehp.123-A64 PMID: 25730842

59. Chesler EJ, Gatti DM, Morgan AP, Strobel M, Trepanier L, Oberbeck D, et al. Diversity Outbred Mice at 21: Maintaining Allelic Variation in the Face of Selection. G3 (Bethesda). 2016; 6(12):3893-902. https://doi.org/10.1534/g3.116.035527 PMID: 27694113

60. Svenson KL, Gatti DM, Valdar W, Welsh CE, Cheng R, Chesler EJ, et al. High-resolution genetic mapping using the Mouse Diversity outbred population. Genetics. 2012; 190(2):437-47. Epub 2012/02/22. https://doi.org/10.1534/genetics.111.132597 PMID: 22345611

61. Odet F, Pan W, Bell TA, Goodson SG, Stevans AM, Yun Z, et al. The Founder Strains of the Collaborative Cross Express a Complex Combination of Advantageous and Deleterious Traits for Male Reproduction. G3 (Bethesda). 2015; 5(12):2671-83. https://doi.org/10.1534/g3.115.020172 PMID: 26483008

62. Bogue MA, Churchill GA, Chesler EJ. Collaborative Cross and Diversity Outbred data resources in the Mouse Phenome Database. Mammalian genome: official journal of the International Mammalian Genome Society. 2015; 26(9-10):511-20. https://doi.org/10.1007/s00335-015-9595-6 PMID: 26286858

63. Keane TM, Goodstadt L, Danecek P, White MA, Wong K, Yalcin B, et al. Mouse genomic variation and its effect on phenotypes and gene regulation. Nature. 2011; 477(7364):289-94. Epub 2011/09/17. https://doi.org/10.1038/nature10413 PMID: 21921910

64. Threadgill DW, Miller DR, Churchill GA, de Villena FP. The collaborative cross: a recombinant inbred mouse population for the systems genetic era. ILAR journal. 2011; 52(1):24-31. Epub 2011/03/18. https://doi.org/10.1093/ilar.52.1.24 PMID: 21411855.

65. Huang da W, Sherman BT, Lempicki RA. Systematic and integrative analysis of large gene lists using DAVID bioinformatics resources. Nature protocols. 2009; 4(1):44-57. Epub 2009/01/10. https://doi. org/10.1038/nprot.2008.211 PMID: 19131956.

66. Huang da W, Sherman BT, Lempicki RA. Bioinformatics enrichment tools: paths toward the comprehensive functional analysis of large gene lists. Nucleic acids research. 2009; 37(1):1-13. Epub 2008/ 11/27. https://doi.org/10.1093/nar/gkn923 PMID: 19033363

67. Wauthier V, Sugathan A, Meyer RD, Dombkowski AA, Waxman DJ. Intrinsic sex differences in the early growth hormone responsiveness of sex-specific genes in mouse liver. Molecular endocrinology (Baltimore, Md). 2010; 24(3):667-78. Epub 2010/02/13. https://doi.org/10.1210/me.2009-0454 PMID: 20150183

68. Gu T, Gatti DM, Srivastava A, Snyder EM, Raghupathy N, Simecek P, et al. Genetic Architectures of Quantitative Variation in RNA Editing Pathways. Genetics. 2016; 202(2):787-98. Epub 2015/11/29. https://doi.org/10.1534/genetics.115.179481 PMID: 26614740

69. Munger SC, Raghupathy N, Choi K, Simons AK, Gatti DM, Hinerfeld DA, et al. RNA-Seq alignment to individualized genomes improves transcript abundance estimates in multiparent populations. Genetics. 2014; 198(1):59-73. Epub 2014/09/23. https://doi.org/10.1534/genetics.114.165886 PMID: 25236449

70. Kukurba KR, Parsana P, Balliu B, Smith KS, Zappala Z, Knowles DA, et al. Impact of the X Chromosome and sex on regulatory variation. Genome research. 2016; 26(6):768-77. Epub 2016/05/20. https://doi.org/10.1101/gr.197897.115 PMID: 27197214 
71. Sidorenko J, Kassam I, Kemper KE, Zeng J, Lloyd-Jones LR, Montgomery GW, et al. The effect of Xlinked dosage compensation on complex trait variation. Nature communications. 2019; 10(1):3009. Epub 2019/07/10. https://doi.org/10.1038/s41467-019-10598-y PMID: 31285442

72. Matthews BJ, Waxman DJ. Computational prediction of CTCF/cohesin-based intra-TAD loops that insulate chromatin contacts and gene expression in mouse liver. eLife. 2018;7. Epub 2018/05/15. https://doi.org/10.7554/eLife.34077 PMID: 29757144

73. Meyer RD, Laz EV, Su T, Waxman DJ. Male-specific hepatic Bcl6: growth hormone-induced block of transcription elongation in females and binding to target genes inversely coordinated with STAT5. Molecular endocrinology (Baltimore, Md). 2009; 23(11):1914-26. Epub 2009/10/03. https://doi.org/10. 1210/me.2009-0242 PMID: 19797429

74. Zhang D, Utsumi T, Huang HC, Gao L, Sangwung P, Chung C, et al. Reticulon 4B (Nogo-B) is a novel regulator of hepatic fibrosis. Hepatology (Baltimore, Md). 2011; 53(4):1306-15. Epub 2011/04/12. https://doi.org/10.1002/hep.24200 PMID: 21480333

75. Zagory JA, Fenlon M, Dietz W, Zhao M, Nguyen MV, Trinh P, et al. Prominin-1 Promotes Biliary Fibrosis Associated With Biliary Atresia. Hepatology (Baltimore, Md). 2019; 69(6):2586-97. Epub 2019/02/ 07. https://doi.org/10.1002/hep.30550 PMID: 30723921

76. Svenson KL, Von Smith R, Magnani PA, Suetin HR, Paigen B, Naggert JK, et al. Multiple trait measurements in 43 inbred mouse strains capture the phenotypic diversity characteristic of human populations. Journal of applied physiology (Bethesda, Md: 1985). 2007; 102(6):2369-78. Epub 2007/02/24. https://doi.org/10.1152/japplphysiol.01077.2006 PMID: 17317875.

77. Fengler VH, Macheiner T, Kessler SM, Czepukojc B, Gemperlein K, Muller R, et al. Susceptibility of Different Mouse Wild Type Strains to Develop Diet-Induced NAFLD/AFLD-Associated Liver Disease. PloS one. 2016; 11(5):e0155163. Epub 2016/05/12. https://doi.org/10.1371/journal.pone.0155163 PMID: 27167736

78. Hillebrandt S, Wasmuth HE, Weiskirchen R, Hellerbrand C, Keppeler H, Werth A, et al. Complement factor 5 is a quantitative trait gene that modifies liver fibrogenesis in mice and humans. Nature genetics. 2005; 37(8):835-43. Epub 2005/07/05. https://doi.org/10.1038/ng1599 PMID: 15995705.

79. Church RJ, Gatti DM, Urban TJ, Long N, Yang X, Shi Q, et al. Sensitivity to hepatotoxicity due to epigallocatechin gallate is affected by genetic background in diversity outbred mice. Food Chem Toxicol. 2015; 76:19-26. https://doi.org/10.1016/j.fct.2014.11.008 PMID: 25446466

80. Tryndyak V, de Conti A, Kobets T, Kutanzi K, Koturbash I, Han T, et al. Interstrain differences in the severity of liver injury induced by a choline- and folate-deficient diet in mice are associated with dysregulation of genes involved in lipid metabolism. FASEB J. 2012; 26(11):4592-602. https://doi.org/10. 1096/fj.12-209569 PMID: 22872676

81. Bavia L, de Castro IA, Isaac L. C57BL/6 and A/J Mice Have Different Inflammatory Response and Liver Lipid Profile in Experimental Alcoholic Liver Disease. Mediators of inflammation. 2015; 2015:491641. Epub 2015/10/09. https://doi.org/10.1155/2015/491641 PMID: 26448681

82. Takahashi K, Yan I, Haga H, Patel T. Long noncoding RNA in liver diseases. Hepatology (Baltimore, Md). 2014; 60(2):744-53. Epub 2014/02/05. https://doi.org/10.1002/hep.27043 PMID: 24493213

83. Shimizu I, Kohno N, Tamaki K, Shono M, Huang HW, He JH, et al. Female hepatology: favorable role of estrogen in chronic liver disease with hepatitis B virus infection. World J Gastroenterol. 2007; 13 (32):4295-305. Epub 2007/08/22. https://doi.org/10.3748/wjg.v13.i32.4295 PMID: 17708600

84. Ayonrinde OT, Olynyk JK, Beilin LJ, Mori TA, Pennell CE, de Klerk N, et al. Gender-specific differences in adipose distribution and adipocytokines influence adolescent nonalcoholic fatty liver disease. Hepatology (Baltimore, Md). 2011; 53(3):800-9. Epub 2011/03/05. https://doi.org/10.1002/hep.24097 PMID: 21374659.

85. Gonzalez FJ. Role of cytochromes P450 in chemical toxicity and oxidative stress: studies with CYP2E1. Mutation research. 2005; 569(1-2):101-10. https://doi.org/10.1016/j.mrfmmm.2004.04 021 PMID: 15603755.

86. Oyola MG, Zuloaga DG, Carbone D, Malysz AM, Acevedo-Rodriguez A, Handa RJ, et al. CYP7B1 Enzyme Deletion Impairs Reproductive Behaviors in Male Mice. Endocrinology. 2015; 156(6):215061. https://doi.org/10.1210/en.2014-1786 PMID: 25849728

87. Dai D, Mills PB, Footitt E, Gissen P, McClean P, Stahlschmidt J, et al. Liver disease in infancy caused by oxysterol 7 alpha-hydroxylase deficiency: successful treatment with chenodeoxycholic acid. J Inherit Metab Dis. 2014; 37(5):851-61. https://doi.org/10.1007/s10545-014-9695-6 PMID: 24658845.

88. Yetti $\mathrm{H}$, Naito $\mathrm{H}$, Yuan $\mathrm{Y}$, Jia $X$, Hayashi $\mathrm{Y}$, Tamada $\mathrm{H}$, et al. Bile acid detoxifying enzymes limit susceptibility to liver fibrosis in female SHRSP5/Dmcr rats fed with a high-fat-cholesterol diet. PloS one. 2018; 13(2):e0192863. https://doi.org/10.1371/journal.pone.0192863 PMID: 29438418. 
89. Stiles AR, McDonald JG, Bauman DR, Russell DW. CYP7B1: one cytochrome P450, two human genetic diseases, and multiple physiological functions. J Biol Chem. 2009; 284(42):28485-9. https:// doi.org/10.1074/jbc.R109.042168 PMID: 19687010

90. Lake AD, Novak P, Shipkova P, Aranibar N, Robertson D, Reily MD, et al. Decreased hepatotoxic bile acid composition and altered synthesis in progressive human nonalcoholic fatty liver disease. Toxicol Appl Pharmacol. 2013; 268(2):132-40. https://doi.org/10.1016/j.taap.2013.01.022 PMID: 23391614

91. Chella Krishnan K, Kurt Z, Barrere-Cain R, Sabir S, Das A, Floyd R, et al. Integration of Multi-omics Data from Mouse Diversity Panel Highlights Mitochondrial Dysfunction in Non-alcoholic Fatty Liver Disease. Cell Syst. 2018; 6(1):103-15 e7. https://doi.org/10.1016/j.cels.2017.12.006 PMID: 29361464

92. Hashemi M, Eskandari-Nasab E, Fazaeli A, Bahari A, Hashemzehi NA, Shafieipour S, et al. Association of genetic polymorphisms of glutathione-S-transferase genes (GSTT1, GSTM1, and GSTP1) and susceptibility to nonalcoholic fatty liver disease in Zahedan, Southeast Iran. DNA and cell biology. 2012; 31(5):672-7. Epub 2011/10/21. https://doi.org/10.1089/dna.2011.1343 PMID: 22011249.

93. Hori M, Oniki K, Nakagawa T, Takata K, Mihara S, Marubayashi T, et al. Association between combinations of glutathione-S-transferase $\mathrm{M} 1, \mathrm{~T} 1$ and $\mathrm{P} 1$ genotypes and non-alcoholic fatty liver disease. Liver international: official journal of the International Association for the Study of the Liver. 2009; 29 (2):164-8. Epub 2008/05/22. https://doi.org/10.1111/j.1478-3231.2008.01794.x PMID: 18492019.

94. Dragovic S, Venkataraman H, Begheijn S, Vermeulen NP, Commandeur JN. Effect of human glutathione S-transferase hGSTP1-1 polymorphism on the detoxification of reactive metabolites of clozapine, diclofenac and acetaminophen. Toxicology letters. 2014; 224(2):272-81. Epub 2013/11/05. https:// doi.org/10.1016/j.toxlet.2013.10.023 PMID: 24185126.

95. Liang S, Kisseleva T, Brenner DA. The Role of NADPH Oxidases (NOXs) in Liver Fibrosis and the Activation of Myofibroblasts. Front Physiol. 2016; 7:17. https://doi.org/10.3389/fphys.2016.00017 PMID: 26869935

96. Jiang JX, Torok NJ. NADPH Oxidases in Chronic Liver Diseases. Adv Hepatol. 2014; 2014. https:// doi.org/10.1155/2014/742931 PMID: 26436133

97. Bettaieb A, Jiang JX, Sasaki Y, Chao TI, Kiss Z, Chen X, et al. Hepatocyte Nicotinamide Adenine Dinucleotide Phosphate Reduced Oxidase 4 Regulates Stress Signaling, Fibrosis, and Insulin Sensitivity During Development of Steatohepatitis in Mice. Gastroenterology. 2015; 149(2):468-80 e10. https:// doi.org/10.1053/j.gastro.2015.04.009 PMID: 25888330

98. French JE, Gatti DM, Morgan DL, Kissling GE, Shockley KR, Knudsen GA, et al. Diversity Outbred Mice Identify Population-Based Exposure Thresholds and Genetic Factors that Influence BenzeneInduced Genotoxicity. Environ Health Perspect. 2015; 123(3):237-45. https://doi.org/10.1289/ehp. 1408202 PMID: 25376053

99. Dechassa ML, Tryndyak V, de Conti A, Xiao W, Beland FA, Pogribny IP. Identification of chromatinaccessible domains in non-alcoholic steatohepatitis-derived hepatocellular carcinoma. Mol Carcinog. 2018; 57(8):978-87. https://doi.org/10.1002/mc.22818 PMID: 29603380.

100. Kahali B, Halligan B, Speliotes EK. Insights from Genome-Wide Association Analyses of Nonalcoholic Fatty Liver Disease. Semin Liver Dis. 2015; 35(4):375-91. https://doi.org/10.1055/s-0035-1567870 PMID: 26676813

101. Parks BW, Sallam T, Mehrabian M, Psychogios N, Hui ST, Norheim F, et al. Genetic architecture of insulin resistance in the mouse. Cell Metab. 2015; 21(2):334-47. https://doi.org/10.1016/j.cmet.2015. 01.002 PMID: 25651185

102. Farh KK, Marson A, Zhu J, Kleinewietfeld M, Housley WJ, Beik S, et al. Genetic and epigenetic fine mapping of causal autoimmune disease variants. Nature. 2015; 518(7539):337-43. https://doi.org/10. 1038/nature13835 PMID: 25363779

103. Maurano MT, Humbert R, Rynes E, Thurman RE, Haugen E, Wang H, et al. Systematic localization of common disease-associated variation in regulatory DNA. Science. 2012; 337(6099):1190-5. https:// doi.org/10.1126/science.1222794 PMID: 22955828

104. Degner JF, Pai AA, Pique-Regi R, Veyrieras JB, Gaffney DJ, Pickrell JK, et al. DNase I sensitivity QTLs are a major determinant of human expression variation. Nature. 2012; 482(7385):390-4. https:// doi.org/10.1038/nature10808 PMID: 22307276

105. Gusev A, Lee SH, Trynka G, Finucane H, Vilhjalmsson BJ, Xu H, et al. Partitioning heritability of regulatory and cell-type-specific variants across 11 common diseases. Am J Hum Genet. 2014; 95(5):53552. https://doi.org/10.1016/j.ajhg.2014.10.004 PMID: 25439723

106. Krebs CJ, Larkins LK, Khan SM, Robins DM. Expansion and diversification of KRAB zinc-finger genes within a cluster including Regulator of sex-limitation 1 and 2. Genomics. 2005; 85(6):752-61. https:// doi.org/10.1016/j.ygeno.2005.03.004 PMID: 15885501. 
107. Krebs CJ, Larkins LK, Price R, Tullis KM, Miller RD, Robins DM. Regulator of sex-limitation (RsI) encodes a pair of KRAB zinc-finger genes that control sexually dimorphic liver gene expression. Genes \& development. 2003; 17(21):2664-74. https://doi.org/10.1101/gad.1135703 PMID: 14563677

108. Rinn JL, Chang HY. Long Noncoding RNAs: Molecular Modalities to Organismal Functions. Annual review of biochemistry. 2020; 89:283-308. Epub 2020/06/23. https://doi.org/10.1146/annurevbiochem-062917-012708 PMID: 32569523.

109. Long $Y$, Wang $X$, Youmans DT, Cech TR. How do IncRNAs regulate transcription? Science advances. 2017; 3(9):eaao2110. Epub 2017/09/30. https://doi.org/10.1126/sciadv.aao2110 PMID: 28959731

110. Kassam I, Lloyd-Jones L, Holloway A, Small KS, Zeng B, Bakshi A, et al. Autosomal genetic control of human gene expression does not differ across the sexes. Genome biology. 2016; 17(1):248. Epub 2016/12/03. https://doi.org/10.1186/s13059-016-1111-0 PMID: 27908293

111. Collaborative Cross $C$. The genome architecture of the Collaborative Cross mouse genetic reference population. Genetics. 2012; 190(2):389-401. Epub 2012/02/22. https://doi.org/10.1534/genetics.111. 132639 PMID: 22345608

112. Morgan AP, Fu CP, Kao CY, Welsh CE, Didion JP, Yadgary L, et al. The Mouse Universal Genotyping Array: From Substrains to Subspecies. G3 (Bethesda). 2015; 6(2):263-79. Epub 2015/12/20. https:// doi.org/10.1534/g3.115.022087 PMID: 26684931

113. Gatti DM, Svenson KL, Shabalin A, Wu LY, Valdar W, Simecek $P$, et al. Quantitative trait locus mapping methods for diversity outbred mice. G3 (Bethesda). 2014; 4(9):1623-33. https://doi.org/10.1534/ g3.114.013748 PMID: 25237114

114. Kim D, Pertea G, Trapnell C, Pimentel H, Kelley R, Salzberg SL. TopHat2: accurate alignment of transcriptomes in the presence of insertions, deletions and gene fusions. Genome biology. 2013; 14(4): R36. Epub 2013/04/27. https://doi.org/10.1186/gb-2013-14-4-r36 PMID: 23618408

115. Langmead B, Trapnell C, Pop M, Salzberg SL. Ultrafast and memory-efficient alignment of short DNA sequences to the human genome. Genome biology. 2009; 10(3):R25. Epub 2009/03/06. https://doi. org/10.1186/gb-2009-10-3-r25 PMID: 19261174

116. Liao Y, Smyth GK, Shi W. featureCounts: an efficient general purpose program for assigning sequence reads to genomic features. Bioinformatics. 2014; 30(7):923-30. Epub 2013/11/15. https:// doi.org/10.1093/bioinformatics/btt656 PMID: 24227677.

117. Robinson MD, McCarthy DJ, Smyth GK. edgeR: a Bioconductor package for differential expression analysis of digital gene expression data. Bioinformatics. 2010; 26(1):139-40. Epub 2009/11/17. https://doi.org/10.1093/bioinformatics/btp616 PMID: 19910308

118. Smyth GK. Linear models and empirical bayes methods for assessing differential expression in microarray experiments. Statistical applications in genetics and molecular biology. 2004; 3:Article3. Epub 2006/05/02. https://doi.org/10.2202/1544-6115.1027 PMID: 16646809.

119. Leek JT, Johnson WE, Parker HS, Jaffe AE, Storey JD. The sva package for removing batch effects and other unwanted variation in high-throughput experiments. Bioinformatics. 2012; 28(6):882-3. Epub 2012/01/20. https://doi.org/10.1093/bioinformatics/bts034 PMID: 22257669

120. Rosenbloom KR, Armstrong J, Barber GP, Casper J, Clawson H, Diekhans M, et al. The UCSC Genome Browser database: 2015 update. Nucleic acids research. 2015; 43(Database issue):D67081. Epub 2014/11/28. https://doi.org/10.1093/nar/gku1177 PMID: 25428374

121. Mudunuri U, Che A, Yi M, Stephens RM. bioDBnet: the biological database network. Bioinformatics. 2009; 25(4):555-6. Epub 2009/01/09. https://doi.org/10.1093/bioinformatics/btn654 PMID: 19129209 